\title{
Quantitative Refinement for Weighted Modal Transition Systems
}

\author{
Sebastian S. Bauer ${ }^{1}$, Uli Fahrenberg ${ }^{2}$, Line Juhl ${ }^{3}$, \\ Kim G. Larsen ${ }^{3}$, Axel Legay ${ }^{2}$, and Claus Thrane ${ }^{3}$ \\ 1 Ludwig-Maximilians-Universität München, Germany \\ 2 Irisa/INRIA Rennes, France \\ 3 Aalborg University, Denmark
}

\begin{abstract}
Specification theories as a tool in the development process of component-based software systems have recently attracted a considerable attention. Current specification theories are however qualitative in nature and hence fragile and unsuited for modern software systems. We propose the first specification theory which allows to capture quantitative aspects during the refinement and implementation process.
\end{abstract}

Keywords: reducing complexity of design, modal specifications, quantitative reasoning

\section{Introduction}

Rigorous design of modern computer systems faces the major challenge that the systems are too complex to reason about [16]. Hence it is necessary to reason at the level of specification rather than at the one of implementations. Such specifications, which act as finite and concise abstractions for possibly infinite sets of implementations, allow not only to decrease the complexity of the design, but also permit to reason on subsystems independently.

Any reasonable specification theory is equipped with a satisfaction relation to decide whether an implementation matches the requirements of a specification, and a refinement relation that allows to compare specifications (hence sets of implementations). Moreover, the theory needs a notion of logical composition which allows to infer larger specifications as logical combinations of smaller ones. Another important ingredient is a notion of structural composition that allows to build overall specifications from sub-specifications, mimicking at the implementation level e.g. the interaction of components in a distributed system. A partial inverse of this operation is given by the notion of quotient which allows to synthesize a sub-specification from an overall specification and an implementation which realizes a part of the overall specification.

Over the years, there have been a series of advances on specification theories $[2,14,5,13]$. The predominant approaches are based on modal logics and process algebras but have the drawback that they cannot naturally embed both logical and structural composition within the same formalism. Moreover, such 
formalisms do not permit to reason from specification to implementation through stepwise refinement.

In order to leverage those problems, the concept of modal transition systems was introduced [12]. In short, modal transition systems are labeled transition systems equipped with two types of transitions: must transitions which are mandatory for any implementation, and may transitions which are optional for implementations. It is well admitted that modal transition systems match all the requirements of a reasonable specification theory (see e.g. [15] for motivations). Also, practical experience shows that the formalism is expressive enough to handle complex industrial problems $[6,17]$.

In a series of recent work $[3,10]$, the modal transition system framework has been extended in order to reason on quantitative aspects, hence providing a new specification theory for more elaborated structures, with the objective to better meet practical needs. In this quantitative setting however, the standard Boolean satisfaction and refinement relations are too fragile. Indeed, either an implementation satisfies a specification or it does not. This means that minor and major modifications in the implementation cannot be distinguished, as both of them may reverse the Boolean answer. As observed by de Alfaro et al. for the logical framework of CTL [1], this view is obsolete; engineers need quantitative notions on how modified implementations differ.

The main contribution of this paper is to mitigate the above problem by lifting the satisfaction and refinement relations into the quantitative framework, hence completing the quantitative approach to reason on modal transition systems. More precisely, and similarly to what has been proposed in the logical framework, we introduce a notion of distance between both specifications and implementations, which permits quantitative comparison. Given two implementations that do not necessarily satisfy a specification, we can decide through quantitative reasoning which one is the better match for the specification's requirements.

To facilitate this reasoning, we develop a notion of modal distance between specifications, which approximates the distances between their implementations. This preserves the relation between modal refinement and satisfaction checking in the Boolean setting. We show that computing distances between implementation sets is ExPTIME-hard, whereas modal distances are computable in $\mathrm{NP} \cap \mathrm{CO}-\mathrm{NP}$ (which is higher than for Boolean modal refinement). Akin to discounted games [19] we can reason on behaviors in a discounted manner, giving more importance to differences that happen in the near future, while accumulating the amount by which the specifications fail to be compatible at each step. As for the games, the semantics of the outcome is considered application specific.

Modifying the semantic outcome of satisfaction has strong impact on operations between specifications. As a second contribution of this paper, we propose quantitative versions of structural composition and quotient which inherit the good properties from the Boolean setting. We also propose a new notion of relaxation, which is inherent to the quantitative framework and allows e.g. to calibrate the quotient operator. 
However, there is no free lunch, and working with distances has a price: some of the properties of logical conjunction and determinization are not preserved in the quantitative setting. More precisely, conjunction is not the greatest lower bound with respect to refinement distance as it is in the Boolean setting, and deterministic overapproximation is too coarse. In fact we show that this is a fundamental limitation of any reasonable quantitative specification formalism.

Structure of the paper. We start out by introducing our quantitative formalism which has weighted transition systems as implementations and weighted modal transition systems as specifications. In Section 3 we introduce the distances we use for quantitative comparison of both implementations and specification. Section 4 is devoted to a formalization of the notion of relaxation which is of great use in quantitative design. In the next section we see some inherent limitations of the quantitative approach, and Section 6 finishes the paper by showing that structural composition works as expected in the quantitative framework and links relaxation to quotients. Note that proofs of all theorems in the paper can be found in an appendix.

Acknowledgment. The authors wish to thank Jiří Srba for fruitful discussions during the preparation of this work.

\section{Weighted Modal Transition Systems}

In this section we present the formalism we use for implementations and specifications. As implementations we choose the model of weighted transition systems, i.e. labeled transition systems with integer weights at transitions. Specifications both have a modal dimension, specifying discrete behavior which must be implemented and behavior which may be present in implementations, and a quantitative dimension, specifying intervals of weights on each transition which an implementation must choose from.

Let $\mathbb{I}=\{[x, y] \mid x \in \mathbb{Z} \cup\{-\infty\}, y \in \mathbb{Z} \cup\{\infty\}, x \leq y\}$ be the set of closed extended-integer intervals and let $\Sigma$ be a finite set of actions. Our set of specification labels is Spec $=(\Sigma \times \mathbb{I}) \cup\{\perp\}$, where the special symbol $\perp$ models inconsistency. The set of implementation labels is defined as $\operatorname{Imp}=\Sigma \times\{[x, x] \mid$ $x \in \mathbb{Z}\} \approx \Sigma \times \mathbb{Z}$. Hence a specification imposes labels and integer intervals which constrain the possible weights of an implementation.

We define a partial order on $\mathbb{I}$ (representing inclusion of intervals) by $[x, y] \sqsubseteq$ $\left[x^{\prime}, y^{\prime}\right]$ if $x^{\prime} \leq x$ and $y \leq y^{\prime}$, and we extend this order to specification labels by $(a, I) \sqsubseteq\left(a^{\prime}, I^{\prime}\right)$ if $a=a^{\prime}$ and $I \sqsubseteq I^{\prime}$, and $\perp \sqsubseteq(a, I)$ for all $(a, I) \in$ Spec. The partial order on Spec is hence a refinement order; if $k_{1} \sqsubseteq k_{2}$, then no more implementation labels are contained in $k_{1}$ than in $k_{2}$.

Specifications and implementations are defined as follows:

Definition 1. A weighted modal transition system (WMTS) is a four-tuple $\left(S, s^{0},-\rightarrow, \longrightarrow\right)$ consisting of a set of states $S$ with an initial state $s^{0} \in S$ and may and must transition relations satisfying $\longrightarrow \subseteq-\rightarrow \subseteq S \times$ Spec $\times S$. A WMTS is an implementation if $\longrightarrow=-\rightarrow \subseteq S \times \operatorname{Imp} \times S$. 
A WMTS is finite if $S$ and $\rightarrow$ (and hence also $\longrightarrow$ ) are finite sets, and it is deterministic if it holds that for any $s \in S$ and $a \in \Sigma,\left(s,\left(a, I_{1}\right), t_{1}\right),\left(s,\left(a, I_{2}\right), t_{2}\right) \in$ $\rightarrow$ imply $I_{1}=I_{2}$ and $t_{1}=t_{2}$. Hence a deterministic specification allows at most one transition under each discrete action from every state. In the rest of the paper we will write $s-\stackrel{k}{\rightarrow} s^{\prime}$ for $\left(s, k, s^{\prime}\right) \in \rightarrow$ and similarly for $\longrightarrow$, and we will always write $S=\left(S, s^{0},-\rightarrow, \longrightarrow\right)$ or $S_{i}=\left(S_{i}, s_{i}^{0},-\rightarrow_{i}, \longrightarrow_{i}\right)$ for WMTS and $I=\left(I, i^{0}, \longrightarrow\right)$ for implementations.

The implementation semantics of a specification is given through modal refinement, as follows: A modal refinement of WMTS $S_{1}, S_{2}$ is a relation $R \subseteq$ $S_{1} \times S_{2}$ such that for any $\left(s_{1}, s_{2}\right) \in R$ and any may transition $s_{1}-_{-{ }_{1} \rightarrow_{1}} t_{1}$ in $S_{1}$, there exists $s_{2} \stackrel{k_{2}}{\rightarrow}{ }_{2} t_{2}$ in $S_{2}$ for which $k_{1} \sqsubseteq k_{2}$ and $\left(t_{1}, t_{2}\right) \in R$, and for any must transition $s_{2} \stackrel{k_{2}}{\longrightarrow} t_{2}$ in $S_{2}$, there exists $s_{1} \stackrel{k_{1}}{\longrightarrow} t_{1}$ in $S_{1}$ for which $k_{1} \sqsubseteq k_{2}$ and $\left(t_{1}, t_{2}\right) \in R$. Hence in such a modal refinement, behavior which is required in $S_{2}$ is also required in $S_{1}$, no more behavior is allowed in $S_{1}$ than in $S_{2}$, and the quantitative requirements in $S_{1}$ are refinements of the ones in $S_{2}$. We write $S_{1} \leq_{m} S_{2}$ if there is a modal refinement relation $R$ for which $\left(s_{1}^{0}, s_{2}^{0}\right) \in R$. The implementation semantics of a specification can then be defined as the set of all implementations which are also refinements:

Definition 2. The implementation semantics of a WMTS $S$ is the set $\llbracket S \rrbracket=$ $\left\{I \mid I \leq_{m} S, I\right.$ implementation $\}$.

We say that a WMTS $S$ is consistent if it has an implementation, i.e. if $\llbracket S \rrbracket \neq$ $\emptyset$. A useful over-approximation of consistency is local consistency: a WMTS $S$ is said to be locally consistent if $s \stackrel{k}{\longrightarrow} t$ implies $k \neq \perp$, i.e. if no $\perp$-labeled must transitions appear in $S$. Local consistency implies consistency, but the inverse is not true; e.g. the WMTS $s_{0} \stackrel{a, 2}{\longrightarrow} s_{1} \stackrel{a, 9}{\rightarrow} s_{2} \stackrel{\perp}{\longrightarrow} s_{3}$ has an implementation $i_{0} \stackrel{a, 2}{\longrightarrow} i_{1}$. Local inconsistencies may be removed recursively as follows:

Definition 3. For a WMTS $S$, let pre $: 2^{S} \rightarrow 2^{S}$ be given by pre(B) $=\{s \in$ $S \mid s \stackrel{k}{\longrightarrow} t \in B$ for some $k\}$, and let $S^{\perp}=\{s \in S \mid s \stackrel{\perp}{\longrightarrow} t$ for some $t \in S\}$. If $s^{0} \notin \operatorname{pre}^{*}\left(S^{\perp}\right)$, then the pruning $\rho(S)=\left(S_{\rho}, s^{0},-\rightarrow \rho, \longrightarrow_{\rho}\right)$ is defined by $S_{\rho}=S \backslash \operatorname{pre}^{*}\left(S^{\perp}\right),-\rightarrow_{\rho}=-\rightarrow \cap\left(S_{\rho} \times(\right.$ Spec $\left.\backslash\{\perp\}) \times S_{\rho}\right)$ and $\longrightarrow \rho=\longrightarrow \cap$ $\left(S_{\rho} \times(\operatorname{Spec} \backslash\{\perp\}) \times S_{\rho}\right)$.

Note that if $\rho(S)$ exists, then it is locally consistent, and if $\rho(S)$ does not exist $\left(s^{0} \in \operatorname{pre}^{*}\left(S^{\perp}\right)\right)$, then $S$ is inconsistent. Also, $\rho(S) \leq_{m} S$ and $\llbracket \rho(S) \rrbracket=\llbracket S \rrbracket$.

\section{Thorough and Modal Refinement Distances}

For the quantitative specification formalism we have introduced in the last section, the standard Boolean notions of satisfaction and refinement are too fragile. To be able to reason not only whether a given quantitative implementation satisfies a given quantitative specification, but also to what extent, we introduce a notion of distance between both implementations and specifications. 


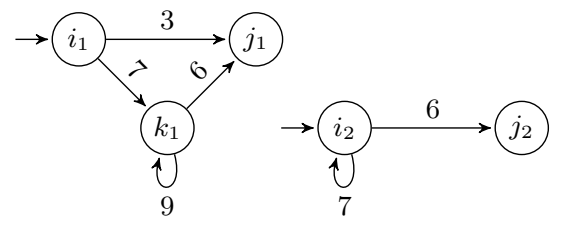

$$
\begin{aligned}
& d\left(j_{1}, j_{2}\right)=0 \\
& d\left(i_{1}, j_{2}\right)=\infty \\
& d\left(j_{1}, i_{2}\right)=\infty \\
& d\left(k_{1}, j_{2}\right)=\infty \\
& d\left(k_{1}, i_{2}\right)=\max \{2+.9 d\left(k_{1}, i_{2}\right), .9 \overbrace{d\left(j_{1}, j_{2}\right)}^{0}\} \\
& d\left(i_{1}, i_{2}\right)=\max \{3+.9 \underbrace{d\left(j_{1}, j_{2}\right)}_{0}, .9 d\left(k_{1}, i_{2}\right)\}
\end{aligned}
$$

Fig. 1. Two weighted transition systems with branching distance $d\left(I_{1}, I_{2}\right)=18$.

We first define the distance between implementations; for this we introduce a distance on implementation labels by

$$
d_{\operatorname{Imp}}\left(\left(a_{1}, x_{1}\right),\left(a_{2}, x_{2}\right)\right)=\left\{\begin{array}{cl}
\infty & \text { if } a_{1} \neq a_{2}, \\
\left|x_{1}-x_{2}\right| & \text { if } a_{1}=a_{2} .
\end{array}\right.
$$

In the rest of the paper, let $\lambda \in \mathbb{R}$ with $0<\lambda<1$ be a discounting factor.

Definition 4. Let $I_{1}, I_{2}$ be implementations (weighted transition systems). The implementation distance $d: I_{1} \times I_{2} \rightarrow \mathbb{R}_{>0} \cup\{\infty\}$ between the states of $I_{1}$ and $I_{2}$ is the least fixed point of the equations

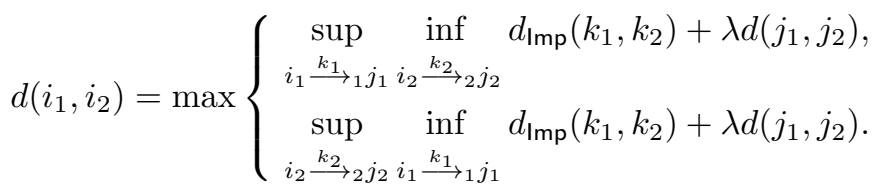

We define $d\left(I_{1}, I_{2}\right)=d\left(i_{1}^{0}, i_{2}^{0}\right)$.

Except for the symmetrizing max operation, this is precisely the accumulating branching distance which is introduced in [18]; see also [8,9] for a thorough introduction to linear and branching distances as we use them here. As the equations in the definition define a contraction, they have indeed a unique least fixed point; note that $d\left(i_{1}, i_{2}\right)=\infty$ is also a fixed point, $c f$. [11].

We remark that besides this accumulating distance, other interesting system distances may be defined depending on the application at hand, but we concentrate here on this distance and leave a generalization to other distances for future work.

Example 1. Consider the two implementations $I_{1}$ and $I_{2}$ in Figure 1 with a single action (elided for simplicity) and with discounting factor $\lambda=.9$. The equations in the illustration have already been simplified by removing all expressions that evaluate to $\infty$. What remains to be done is to compute the least fixed point of the equation $d\left(k_{1}, i_{2}\right)=\max \left\{2+.9 d\left(k_{1}, i_{2}\right), 0\right\}$ which is $d\left(k_{1}, i_{2}\right)=20$. Hence $d\left(i_{1}, i_{2}\right)=\max \{3, .9 \cdot 20\}=18$.

To lift implementation distance to specifications, we need first to consider the distance between sets of implementations. Given implementation sets $\mathcal{I}_{1}, \mathcal{I}_{2}$, we define

$$
d\left(\mathcal{I}_{1}, \mathcal{I}_{2}\right)=\sup _{I_{1} \in \mathcal{I}_{1}} \inf _{I_{2} \in \mathcal{I}_{2}} d\left(I_{1}, I_{2}\right)
$$


Note that in case $\mathcal{I}_{2}$ is finite, we have that for all $\varepsilon \geq 0, d\left(\mathcal{I}_{1}, \mathcal{I}_{2}\right) \leq \varepsilon$ if and only if for each implementation $I_{1} \in \mathcal{I}_{1}$ there exists $I_{2} \in \mathcal{I}_{2}$ for which $d\left(I_{1}, I_{2}\right) \leq \varepsilon$, hence this is a natural notion of distance. Especially, $d\left(\mathcal{I}_{1}, \mathcal{I}_{2}\right)=0$ if and only if $\mathcal{I}_{1}$ is a subset of $\mathcal{I}_{2}$ up to bisimilarity. For infinite $\mathcal{I}_{2}$, we have the slightly more complicated property that $d\left(\mathcal{I}_{1}, \mathcal{I}_{2}\right) \leq \varepsilon$ if and only if for all $\delta>0$ and any $I_{1} \in \mathcal{I}_{1}$, there is $I_{2} \in \mathcal{I}_{2}$ for which $d\left(I_{1}, I_{2}\right) \leq \varepsilon+\delta$.

Note that in general, our distance on sets of implementations is asymmetric; we may well have $d\left(\mathcal{I}_{1}, \mathcal{I}_{2}\right) \neq d\left(\mathcal{I}_{2}, \mathcal{I}_{1}\right)$. We lift this distance to specifications as follows:

Definition 5. The thorough refinement distance between WMTS $S_{1}$ and $S_{2}$ is defined as $d_{t}\left(S_{1}, S_{2}\right)=d\left(\llbracket S_{1} \rrbracket, \llbracket S_{2} \rrbracket\right)$. We write $S_{1} \leq_{t}^{\varepsilon} S_{2}$ if $d_{t}\left(S_{1}, S_{2}\right) \leq \varepsilon$.

Indeed this permits us to measure incompatibility of specifications. Also observe the special case where $S_{1}=I_{1}$ is an implementation: then $d_{t}\left(I_{1}, S_{2}\right)=$ $\inf _{I_{2} \in \llbracket S_{2} \rrbracket} d\left(I_{1}, I_{2}\right)$, which measures how close $I_{1}$ is to satisfy the specification $S_{2}$.

To facilitate computation and comparison of refinement distance, we introduce modal refinement distance as an overapproximation. We will show in Theorem 2 below that similarly to the Boolean setting [4], computation of thorough refinement distance is EXPTIME-hard, whereas modal refinement distance is computable in NP $\cap$ CO-NP. First we generalize the distance on implementation labels from Equation (1) to specification labels so that for $k, \ell \in \mathrm{Spec}$ we define

$$
d_{\text {Spec }}(k, \ell)=\sup _{k^{\prime} \in \llbracket k \rrbracket} \inf _{\ell^{\prime} \in \llbracket \ell \rrbracket} d_{\operatorname{lmp}}\left(k^{\prime}, \ell^{\prime}\right) .
$$

Note that $d_{\mathrm{Spec}}$ is asymmetric, and that $d_{\mathrm{Spec}}(k, \ell)=0$ if and only if $k \sqsubseteq \ell$. Also, $d_{\text {Spec }}(k, \ell)=d_{\operatorname{lmp}}(k, \ell)$ for all $k, \ell \in \operatorname{Imp}$. Using the $\dot{-}$ operation defined on integers by $x_{1} \dot{-} x_{2}=\max \left(x_{1}-x_{2}, 0\right)$, we can express $d_{\text {Spec }}$ as follows:

$$
\begin{aligned}
d_{\text {Spec }}\left(\left(a_{1}, I_{1}\right),\left(a_{2}, I_{2}\right)\right) & =\infty \quad \text { if } a_{1} \neq a_{2} \\
d_{\text {Spec }}\left(\left(a,\left[x_{1}, y_{1}\right]\right),\left(a,\left[x_{2}, y_{2}\right]\right)\right) & =\max \left(x_{2} \dot{-} x_{1}, y_{1} \dot{-} y_{2}\right) \\
d_{\text {Spec }}\left(\perp,\left(a, I_{2}\right)\right)=0 & d_{\text {Spec }}\left(\left(a, I_{1}\right), \perp\right)=\infty
\end{aligned}
$$

Definition 6. Let $S_{1}, S_{2}$ be WMTS. The modal refinement distance $d_{m}: S_{1} \times$ $S_{2} \rightarrow \mathbb{R}_{\geq 0} \cup\{\infty\}$ from states of $S_{1}$ to states of $S_{2}$ is the least fixed point of the equations

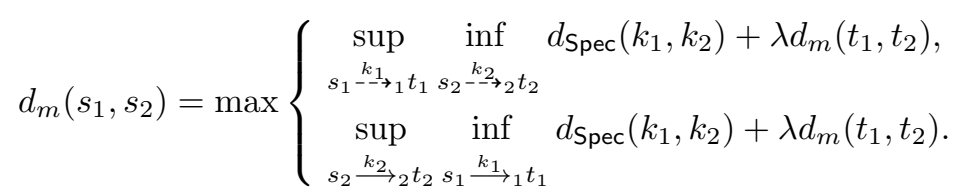

We define $d_{m}\left(S_{1}, S_{2}\right)=d_{m}\left(s_{1}^{0}, s_{2}^{0}\right)$, and we write $S_{1} \leq_{m}^{\varepsilon} S_{2}$ if $d_{m}\left(S_{1}, S_{2}\right) \leq \varepsilon$.

The argument for existence and uniqueness of the least fixed point is exactly the same as for implementation distance in Definition 4. Like thorough refinement distance, modal refinement distance may be asymmetric. 


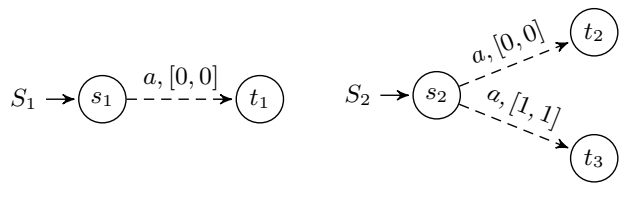

Fig. 2. Incompleteness of modal refinement distance.

The next theorem shows that modal refinement distance indeed overapproximates thorough refinement distance, and that it is exact for deterministic WMTS. Note that nothing general can be said about the precision of the overapproximation in the nondeterministic case; as an example observe the two specifications in Figure 2 for which $d_{t}\left(S_{1}, S_{2}\right)=0$ but $d_{m}\left(S_{1}, S_{2}\right)=\infty$.

Theorem 1. For WMTS $S_{1}, S_{2}$ we have $d_{t}\left(S_{1}, S_{2}\right) \leq d_{m}\left(S_{1}, S_{2}\right)$. If $S_{1}$ is locally consistent and $S_{2}$ is deterministic, then $d_{t}\left(S_{1}, S_{2}\right)=d_{m}\left(S_{1}, S_{2}\right)$.

The complexity results in the next theorem show that modal refinement distance can serve as a useful approximation of thorough refinement distance.

Theorem 2. For finite WMTS $S_{1}, S_{2}$ and $\varepsilon \geq 0$, it is ExPTIME-hard to decide whether $S_{1} \leq_{t}^{\varepsilon} S_{2}$. The problem whether $S_{1} \leq_{m}^{\varepsilon} S_{2}$ is decidable in NP $\cap$ CO-NP.

\section{Relaxation}

We introduce here a notion of relaxation which is specific to the quantitative setting. Intuitively, relaxing a specification means to weaken the quantitative constraints, while the discrete demands on which transitions may or must be present in implementations are kept. A similar notion of strengthening may be defined, but we do not use this here.

Definition 7. For WMTS $S, S^{\prime}$ and $\varepsilon \geq 0, S^{\prime}$ is an $\varepsilon$-relaxation of $S$ if $S \leq_{m} S^{\prime}$ and $S^{\prime} \leq_{m}^{\varepsilon} S$.

Hence the quantitative constraints in $S^{\prime}$ may be more permissive than the ones in $S$, but no new discrete behavior may be introduced. Also note that any implementation of $S$ is also an implementation of $S^{\prime}$, and no implementation of $S^{\prime}$ is further than $\varepsilon$ away from an implementation of $S$. The following proposition relates specifications to relaxed specifications:

Proposition 1. If $S_{1}^{\prime}, S_{2}^{\prime}$ are $\varepsilon$-relaxations of $S_{1}$ and $S_{2}$, then $d_{m}\left(S_{1}, S_{2}\right)-\varepsilon \leq$ $d_{m}\left(S_{1}, S_{2}^{\prime}\right) \leq d_{m}\left(S_{1}, S_{2}\right)$ and $d_{m}\left(S_{1}, S_{2}\right) \leq d_{m}\left(S_{1}^{\prime}, S_{2}\right) \leq d_{m}\left(S_{1}, S_{2}\right)+\varepsilon$.

On the syntactic level, we can introduce the following widening operator which relaxes all quantitative constraints in a systematic manner. We write $I \pm$ $\delta=[x-\delta, y+\delta]$ for an interval $I=[x, y]$ and $\delta \in \mathbb{N}$. 
Definition 8. Given $\delta \in \mathbb{N}$, the $\delta$-widening of a WMTS $S$ is the WMTS $S^{+\delta}$ with transitions $s \stackrel{a, I \pm \delta}{\rightarrow} t$ in $S^{+\delta}$ for all $s \stackrel{a, I}{\rightarrow} t$ in $S$, and $s \stackrel{a, I \pm \delta}{\longrightarrow} t$ in $S^{+\delta}$ for all $s \stackrel{a, I}{\longrightarrow} t$ in $S$.

Widening and relaxation are related as follows; note also that as widening is a global operation whereas relaxation may be achieved entirely locally, not all relaxations may be obtained as widenings.

Proposition 2. The $\delta$-widening of any WMTS $S$ is a $(1-\lambda)^{-1} \delta$-relaxation.

There is also an implementation-level notion which corresponds to relaxation:

Definition 9. The $\varepsilon$-extended implementation semantics, for $\varepsilon \geq 0$, of a WMTS $S$ is $\llbracket S \rrbracket^{+\varepsilon}=\left\{I \mid I \leq_{m}^{\varepsilon} S, I\right.$ implementation $\}$.

Proposition 3. If $S^{\prime}$ is an $\varepsilon$-relaxation of $S$, then $\llbracket S^{\prime} \rrbracket \subseteq \llbracket S \rrbracket^{+\varepsilon}$.

It can be shown that there are WMTS $S, S^{\prime}$ such that $S^{\prime}$ is an $\varepsilon$-relaxation of $S$ but the inclusion $\llbracket S^{\prime} \rrbracket \subseteq \llbracket S \rrbracket^{+\varepsilon}$ is strict.

\section{Limitations of the Quantitative Approach}

In this section we turn our attention towards some of the standard operators for specification theories; determinization and logical conjunction. Quite surprisingly, we show that in the quantitative setting, there are problems with these notions which do not appear in the Boolean theory. More specifically, we show that there is no determinization operator which always yields a smallest deterministic overapproximation, and there is no conjunction operator which acts as a greatest lower bound.

Theorem 3. There is no unary operator $\mathcal{D}$ on WMTS for which it holds that

(3.1) $\mathcal{D}(S)$ is deterministic for any WMTS $S$,

(3.2) $S \leq_{m} \mathcal{D}(S)$ for any WMTS $S$,

(3.3) $S \leq_{m}^{\varepsilon} D$ implies $\mathcal{D}(S) \leq_{m}^{\varepsilon} D$ for any WMTS $S$, any deterministic WMTS $D$, and any $\varepsilon \geq 0$.

In the standard Boolean setting, there is indeed a determinization operator which satisfies properties similar to the above, and which is useful because it enables checking thorough refinement, $c f$. Theorem 1. Likewise, the greatestlower-bound property of logical conjunction in the Boolean setting ensures that the set of implementations of a conjunction of specifications is precisely the intersection of the implementation sets of the two specifications.

Theorem 4. There is no partial binary operator $\wedge$ on WMTS for which it holds that

(4.1) $S_{1} \wedge S_{2} \leq_{m} S_{1}$ and $S_{1} \wedge S_{2} \leq_{m} S_{2}$ for all locally consistent WMTS $S_{1}, S_{2}$ for which $S_{1} \wedge S_{2}$ is defined, 
(4.2) for any locally consistent WMTS $S$ and all deterministic and locally consistent WMTS $S_{1}, S_{2}$ such that $S \leq_{m} S_{1}$ and $S \leq_{m} S_{2}, S_{1} \wedge S_{2}$ is defined and $S \leq_{m} S_{1} \wedge S_{2}$,

(4.3) for any $\varepsilon \geq 0$, there exist $\varepsilon_{1} \geq 0$ and $\varepsilon_{2} \geq 0$ such that for any locally consistent WMTS $S$ and all deterministic and locally consistent WMTS $S_{1}$, $S_{2}$ for which $S_{1} \wedge S_{2}$ is defined, $S \leq_{m}^{\varepsilon_{1}} S_{1}$ and $S \leq_{m}^{\varepsilon_{2}} S_{2}$ imply $S \leq_{m}^{\varepsilon} S_{1} \wedge S_{2}$.

The counterexamples used in the proofs of Theorems 3 and 4 are quite general and apply to a large class of distances, rather than only to the accumulating distance discussed in this paper. More precisely, it can be shown that Theorem 3 holds for any modal distance $d_{m}$ for which there are label sequences $\left(k_{1}^{1}, k_{1}^{2}\right)$, $\left(k_{2}^{1}, k_{2}^{2}\right),\left(k_{3}^{1}, k_{3}^{2}\right)$ such that (using the obvious specialization of $d_{m}$ to label sequences and $\sqcap$ as lowest upper bound on $(\mathrm{Spec}, \sqsubseteq))$

$$
\begin{aligned}
\max \left(d_{m}\left(\left(k_{1}^{1}, k_{1}^{2}\right),\left(k_{3}^{1}, k_{3}^{2}\right)\right), d_{m}\left(\left(k_{2}^{1}, k_{2}^{2}\right),\right.\right. & \left.\left.\left(k_{3}^{1}, k_{3}^{2}\right)\right)\right) \\
& \neq d_{m}\left(\left(k_{1}^{1} \sqcap k_{2}^{1}, k_{3}^{1}\right),\left(k_{1}^{2} \sqcap k_{2}^{2}, k_{3}^{2}\right)\right) .
\end{aligned}
$$

\section{Structural Composition and Quotient}

In this section we show that in our quantitative setting, notions of structural composition and quotient can be defined which obey the properties expected of such operations. In particular, structural composition satisfies independent implementability [2], hence the refinement distance between structural composites can be bounded by the distances between their respective components.

First we define partial synchronization operators $\oplus$ and $\ominus$ on specification labels which will be used for synchronizing transitions. We let $\left(a_{1}, I_{1}\right) \oplus\left(a_{2}, I_{2}\right)$ and $\left(a_{1}, I_{1}\right) \ominus\left(a_{2}, I_{2}\right)$ be undefined if $a_{1} \neq a_{2}$, and otherwise

$$
\begin{aligned}
& \left(a,\left[x_{1}, y_{1}\right]\right) \oplus\left(a,\left[x_{2}, y_{2}\right]\right)=\left(a,\left[x_{1}+x_{2}, y_{1}+y_{2}\right]\right), \\
& \left(a, I_{1}\right) \oplus \perp=\perp \oplus\left(a, I_{2}\right)=\perp \text {; } \\
& \left(a,\left[x_{1}, y_{1}\right]\right) \ominus\left(a,\left[x_{2}, y_{2}\right]\right)=\left\{\begin{array}{cl}
\perp & \text { if } x_{1}-x_{2}>y_{1}-y_{2}, \\
\left(a,\left[x_{1}-x_{2}, y_{1}-y_{2}\right]\right) & \text { if } x_{1}-x_{2} \leq y_{1}-y_{2},
\end{array}\right. \\
& \left(a, I_{1}\right) \ominus \perp=\perp \ominus\left(a, I_{2}\right)=\perp .
\end{aligned}
$$

Note that we use CSP-style synchronization, but other types of synchronization can easily be defined. Also, defining $\oplus$ to add intervals (and $\ominus$ to subtract them) is only one particular choice; depending on the application, one can also e.g. let $\oplus$ be intersection of intervals or some other operation.

Definition 10. Let $S_{1}$ and $S_{2}$ be WMTS. The structural composition of $S_{1}$ and $S_{2}$ is $S_{1} \| S_{2}=\left(S_{1} \times S_{2},\left(s_{1}^{0}, s_{2}^{0}\right)\right.$, Spec, $\left.-\rightarrow, \longrightarrow\right)$ with transitions given as follows:

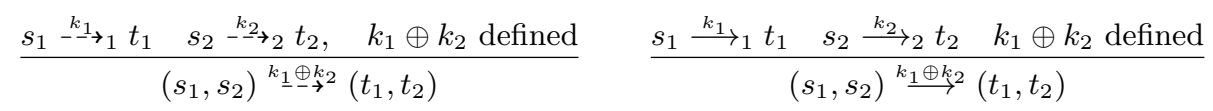




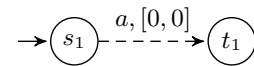

(a) $S_{1}$

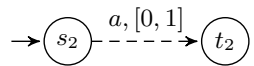

(b) $S_{2}$

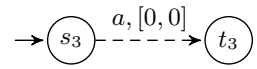

(c) $S_{3}$

$$
\rightarrow\left(s_{2}, s_{3}\right)-a,[0,1] \rightarrow\left(t_{2}, t_{3}\right) \quad \rightarrow\left(s_{1}, s_{2}\right)
$$
(d) $S_{2} \| S_{3}$
(e) $S_{1} \rrbracket S_{2}$

Fig. 3. WMTS for which $d_{m}\left(S_{3}, S_{1} \| S_{2}\right) \neq d_{m}\left(S_{2} \| S_{3}, S_{1}\right)$.

The quotient of $S_{1}$ by $S_{2}$ is $S_{1} \| S_{2}=\rho\left(S_{1} \times S_{2} \cup\{u\},\left(s_{1}^{0}, s_{2}^{0}\right)\right.$, Spec, $\left.-\rightarrow, \longrightarrow\right)$ with transitions given as follows:

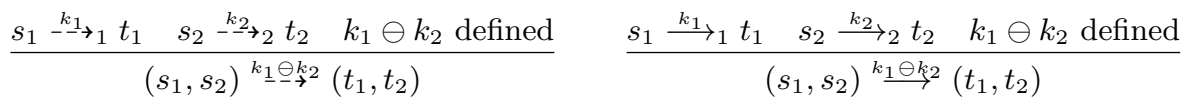

$$
\begin{aligned}
& \frac{s_{1} \stackrel{k_{1}}{\longrightarrow} t_{1} \quad \forall s_{2} \stackrel{k_{2}}{{ }_{2}} t_{2}: k_{1} \ominus k_{2} \text { undefined }}{\left(s_{1}, s_{2}\right) \stackrel{\perp}{\longrightarrow}\left(s_{1}, s_{2}\right)} \\
& \frac{k \in \mathrm{Spec} \forall s_{2} \stackrel{k_{2}}{\rightarrow}{ }_{2} t_{2}: k \oplus k_{2} \text { undefined }}{\left(s_{1}, s_{2}\right)-\stackrel{k}{\rightarrow} u} \quad \frac{k \in \mathrm{Spec}}{u-\stackrel{k}{\rightarrow} u}
\end{aligned}
$$

Note that we ensure that the quotient $S_{1} \| S_{2}$ is locally consistent by recursively removing $\perp$-labeled must transitions using pruning, see Definition 3 . The following theorem shows that structural composition is well-behaved with respect to modal refinement distance in the sense that the distance between the composed systems is bounded by the distances of the individual systems. Note also the special case in the theorem of $S_{1} \leq_{m} S_{2}$ and $S_{3} \leq_{m} S_{4}$ implying $S_{1}\left\|S_{3} \leq_{m} S_{2}\right\| S_{4}$.

Theorem 5 (Independent implementability). For WMTS $S_{1}, S_{2}, S_{3}, S_{4}$ we have $d_{m}\left(S_{1}\left\|S_{3}, S_{2}\right\| S_{4}\right) \leq d_{m}\left(S_{1}, S_{2}\right)+d_{m}\left(S_{3}, S_{4}\right)$.

The following theorem expresses the fact that quotient is a partial inverse to structural composition. Intuitively, the theorem shows that the quotient $S_{1} \| S_{2}$ is maximal among all WMTS $S_{3}$ with respect to any distance $S_{2} \| S_{3} \leq_{m}^{\varepsilon} S_{1}$; note the special case of $S_{3} \leq_{m} S_{1} \| S_{2}$ if and only if $S_{2} \| S_{3} \leq_{m} S_{1}$.

Theorem 6 (Soundness and maximality of quotient). Let $S_{1}, S_{2}$ and $S_{3}$ be locally consistent WMTS such that $S_{2}$ is deterministic and $S_{1} \| S_{2}$ is defined. If $d_{m}\left(S_{3}, S_{1} \| S_{2}\right)<\infty$, then $d_{m}\left(S_{3}, S_{1} \| S_{2}\right)=d_{m}\left(S_{2} \| S_{3}, S_{1}\right)$.

The example depicted in Figure 3 shows that the condition $d_{m}\left(S_{3}, S_{1} \| S_{2}\right)<$ $\infty$ in Theorem 6 is necessary. Here $d_{m}\left(S_{2} \| S_{3}, S_{1}\right)=1$, but $d_{m}\left(S_{3}, S_{1} \| S_{2}\right)=\infty$ because of inconsistency between the transitions $s_{1} \stackrel{\substack{a,[0,0] \\ \rightarrow}}{t_{1}}$ and $s_{2} \stackrel{\substack{a,[0,1] \\ \rightarrow}}{2} t_{2}$ for which $k_{1} \ominus k_{2}$ is defined.

As a practical application, we notice that relaxation as defined in Section 4 can be useful when computing quotients. The quotient construction in Definition 10 introduces local inconsistencies (which afterwards are pruned) whenever 
there is a pair of transitions $s_{1} \stackrel{k_{1}}{\rightarrow}{ }_{1} t_{1}, s_{2} \stackrel{k_{2}}{\rightarrow_{2}} t_{2}$ (or $s_{1} \stackrel{k_{1}}{\longrightarrow} t_{1}, s_{2} \stackrel{k_{2}}{\longrightarrow} t_{2} t_{2}$ ) for which $k_{1} \ominus k_{2}=\perp$. Looking at the definition of $\ominus$, we see that this is the case if $k_{1}=\left(a,\left[x_{1}, y_{1}\right]\right)$ and $k_{2}=\left(a,\left[x_{2}, y_{2}\right]\right)$ are such that $x_{1}-x_{2}>y_{1}-y_{2}$; hence these local inconsistencies can be avoided by enlarging $k_{1}$.

Enlarging quantitative constraints is exactly the intuition of relaxation, thus in practical cases where we get a quotient $S_{1} \| S_{2}$ which is "too inconsistent", we may be able to solve this problem by constructing a suitable $\varepsilon$-relaxation $S_{1}^{\prime}$ of $S_{1}$. Theorems 5 and 6 can then be used to ensure that also $S_{1}^{\prime} \rrbracket S_{2}$ is a relaxation of $S_{1} \rrbracket S_{2}$.

\section{Conclusion and Further Work}

We have shown in this paper that within the quantitative specification framework of weighted modal transition systems, refinement and implementation distances provide a useful tool for robust compositional reasoning. Note that these distances permit us not only to reason about differences between implementations and from implementations to specifications, but they also provide a means by which we can compare specifications directly at the abstract level.

We have shown that for some of the ingredients of our specification theory, namely structural composition and quotient, our formalism is a conservative extension of the standard Boolean notions. We have also noted however, that for determinization and logical conjunction, the properties of the Boolean notions are not preserved, and that this is a fundamental limitation of any reasonable quantitative specification theory. The precise practical implications of this for the applicability of our quantitative specification framework are subject to future work.

\section{References}

1. Luca de Alfaro, Marco Faella, and Mariëlle Stoelinga. Linear and branching system metrics. IEEE Trans. Software Eng., 35(2):258-273, 2009.

2. Luca de Alfaro and Thomas Henzinger. Interface-based design. In Manfred Broy, Johannes Grünbauer, David Harel, and Tony Hoare, editors, Engineering Theories of Software Intensive Systems, volume 195 of NATO Science Series II: Mathematics, Physics and Chemistry, pages 83-104. Springer-Verlag, 2005.

3. Sebastian S. Bauer, Line Juhl, Kim G. Larsen, Axel Legay, and Jiří Srba. Quantitative modal transition systems. 2011. Submitted.

4. Nikola Beneš, Jan Křetínský, Kim G. Larsen, and Jiří Srba. Checking thorough refinement on modal transition systems is EXPTIME-complete. In Martin Leucker and Carroll Morgan, editors, ICTAC, volume 5684 of Lecture Notes in Computer Science, pages 112-126. Springer-Verlag, 2009.

5. Arindam Chakrabarti, Luca de Alfaro, Thomas A. Henzinger, and Freddy Y. C. Mang. Synchronous and bidirectional component interfaces. In Proc. 14th Int. Conference on Computer Aided Verification (CAV), volume 2404 of Lecture Notes in Computer Science, pages 414-427, 2002. 
6. STREP COMBEST (COMponent-Based Embedded Systems design Techniques). http://www. combest.eu/home/.

7. Anne Condon. The complexity of stochastic games. Information and Computation, 96(2):203-224, 1992.

8. Uli Fahrenberg, Kim G. Larsen, and Claus Thrane. A quantitative characterization of weighted Kripke structures in temporal logic. Computing and Informatics, 29(6+):1311-1324, 2010.

9. Uli Fahrenberg, Claus Thrane, and Kim G. Larsen. Distances for weighted transition systems: Games and properties. In Proc. QAPL'11, Electronic Proceedings in Theoretical Computer Science, 2011. To be published.

10. Line Juhl, Kim G. Larsen, and Jiří Srba. Modal transition systems with weight intervals. Journal of Logic and Algebraic Programming, 2011. To be published.

11. Kim G. Larsen, Uli Fahrenberg, and Claus Thrane. Metrics for weighted transition systems: Axiomatization and complexity. Theoretical Computer Science, 2011. 10.1016/j.tcs.2011.04.003.

12. Kim Guldstrand Larsen. Modal specifications. In Automatic Verification Methods for Finite State Systems, volume 407 of Lecture Notes in Computer Science, pages 232-246. Springer, 1989.

13. Nancy Lynch and Mark R. Tuttle. An introduction to input/output automata. CWI-Quarterly, 2(3), 1989.

14. Ulrik Nyman. Modal Transition Systems as the Basis for Interface Theories and Product Lines. PhD thesis, Aalborg University, Department of Computer Science, September 2008.

15. Jean-Baptiste Raclet. Residual for component specifications. Electr. Notes in Theor. Comput. Sci., 215:93-110, 2008.

16. Joseph Sifakis. A vision for computer science - the system perspective. Central European Journal of Computer Science, 1(1):108-116, 2011.

17. SPEEDS (SPEculative and Exploratory Design in Systems Engineering). http: //www.speeds.eu.com.

18. Claus Thrane, Uli Fahrenberg, and Kim G. Larsen. Quantitative simulations of weighted transition systems. Journal of Logic and Algebraic Programming, 79(7):689-703, 2010.

19. Uri Zwick and Mike Paterson. The complexity of mean payoff games. In Ding-Zhu $\mathrm{Du}$ and Ming Li, editors, COCOON, volume 959 of Lecture Notes in Computer Science, pages 1-10. Springer-Verlag, 1995. 


\section{Appendix: Proofs for Section 3}

There is a powerful proof technique introduced for branching distances between implementations in [18] that we here extend to modal refinement distance. We define a modal refinement family as an $\mathbb{R}_{\geq 0}$-indexed family of relations $R=$ $\left\{R_{\varepsilon} \subseteq S_{1} \times S_{2} \mid \varepsilon \geq 0\right\}$ such that for any $\varepsilon$ and any $\left(s_{1}, s_{2}\right) \in R_{\varepsilon}$,

- whenever $s_{1} \stackrel{k_{1}}{\rightarrow} t_{1}$ then $s_{2} \stackrel{-k_{2}}{\rightarrow} t_{2}$ such that $d_{\text {Spec }}\left(k_{1}, k_{2}\right) \leq \varepsilon$ and $\left(t_{1}, t_{2}\right) \in$ $R_{\varepsilon^{\prime}}$ for some $\varepsilon^{\prime} \leq \lambda^{-1}\left(\varepsilon-d_{\text {spec }}\left(k_{1}, k_{2}\right)\right)$,

- whenever $s_{2} \stackrel{k_{2}}{\longrightarrow} t_{2}$ then $s_{1} \stackrel{k_{1}}{\longrightarrow} t_{1}$ such that $d_{\text {Spec }}\left(k_{1}, k_{2}\right) \leq \varepsilon$ and $\left(t_{1}, t_{2}\right) \in$ $R_{\varepsilon^{\prime}}$ for some $\varepsilon^{\prime} \leq \lambda^{-1}\left(\varepsilon-d_{\text {spec }}\left(k_{1}, k_{2}\right)\right)$.

It can then be shown, following the proof strategy in [18] developed for implementations, that $S_{1} \leq_{m}^{\varepsilon} S_{2}$ if and only if there is a modal refinement family $R$ with $\left(s_{1}^{0}, s_{2}^{0}\right) \in R_{\varepsilon} \in R$. Also note that modal refinement families are upward closed in the sense that $\left(s_{1}, s_{2}\right) \in R_{\varepsilon}$ implies that $\left(s_{1}, s_{2}\right) \in R_{\varepsilon^{\prime}}$ for all $\varepsilon^{\prime} \geq \varepsilon$.

Theorem A.1. For WMTS $S_{1}, S_{2}$ we have $d_{t}\left(S_{1}, S_{2}\right) \leq d_{m}\left(S_{1}, S_{2}\right)$.

Proof. If $d_{m}\left(S_{1}, S_{2}\right)=\infty$, we have nothing to prove. Otherwise, let $R=\left\{R_{\varepsilon} \subseteq\right.$ $\left.S_{1} \times S_{2} \mid \varepsilon \geq 0\right\}$ be a modal refinement family which witnesses $d_{m}\left(S_{1}, S_{2}\right)$, i.e. such that $\left(s_{1}^{0}, s_{2}^{0}\right) \in R_{d_{m}\left(S_{1}, S_{2}\right)}$, and let $I_{1} \in \llbracket S_{1} \rrbracket$. We have to expose $I_{2} \in$ $\llbracket S_{2} \rrbracket$ for which $d\left(I_{1}, I_{2}\right) \leq d_{m}\left(S_{1}, S_{2}\right)$.

Let $R^{1} \subseteq I_{1} \times S_{1}$ be a witness for $I_{1} \leq_{m} S_{1}$, define $R_{\varepsilon}^{\prime}=R^{1} \circ R_{\varepsilon} \subseteq I_{1} \times S_{2}$ for all $\varepsilon \geq 0$, and let $R^{\prime}=\left\{R_{\varepsilon}^{\prime} \mid \varepsilon \geq 0\right\}$. The states of $I_{2}=\left(I_{2}, i_{2}^{0}\right.$, Imp, $\left.\longrightarrow I_{2}\right)$ are $I_{2}=S_{2}$ with $i_{2}^{0}=s_{2}^{0}$, and the transitions we define as follows:

For any $i_{1} \stackrel{k_{1}^{\prime}}{\longrightarrow} I_{1} j_{1}$ and any $s_{2} \in S_{2}$ for which $\left(i_{1}, s_{2}\right) \in R_{\varepsilon}^{\prime} \in R^{\prime}$ for some $\varepsilon$, we have $s_{2} \stackrel{k_{2} \rightarrow}{\rightarrow} t_{2}$ with $d_{\text {spec }}\left(k_{1}^{\prime}, k_{2}\right) \leq \varepsilon$ and $\left(j_{1}, t_{2}\right) \in R_{\varepsilon^{\prime}}^{\prime} \in R^{\prime}$ for some $\varepsilon^{\prime} \leq \lambda^{-1}\left(\varepsilon-d_{\text {spec }}\left(k_{1}^{\prime}, k_{2}\right)\right)$. Write $k_{1}^{\prime}=\left(a_{1}^{\prime}, x_{1}^{\prime}\right)$ and $k_{2}=\left(a_{2},\left[x_{2}, y_{2}\right]\right)$, then we must have $a_{1}^{\prime}=a_{2}$. Let

$$
x_{2}^{\prime}= \begin{cases}x_{2} & \text { if } x_{1}^{\prime}<x_{2} \\ x_{1}^{\prime} & \text { if } x_{2} \leq x_{1}^{\prime} \leq y_{2}, \\ y_{2} & \text { if } x_{1}^{\prime}>y_{2}\end{cases}
$$

and $k_{2}^{\prime}=\left(a_{2}, x_{2}^{\prime}\right)$, and put $s_{2} \stackrel{k_{2}^{\prime}}{\longrightarrow} I_{2} t_{2}$ in $I_{2}$. Note that

$$
d_{\text {Spec }}\left(k_{1}^{\prime}, k_{2}^{\prime}\right)=d_{\text {Spec }}\left(k_{1}^{\prime}, k_{2}\right) .
$$

Similarly, for any $s_{2} \stackrel{k_{2}}{\longrightarrow}{ }_{2} t_{2}$ and any $i_{1} \in I_{1}$ with $\left(i_{1}, s_{2}\right) \in R_{\varepsilon}^{\prime} \in R^{\prime}$ for some $\varepsilon$, we have $i_{1} \stackrel{k_{1}^{\prime}}{\longrightarrow} I_{1} j_{1}$ with $d_{\text {Spec }}\left(k_{1}^{\prime}, k_{2}\right) \leq \varepsilon$ and $\left(j_{1}, t_{2}\right) \in R_{\varepsilon^{\prime}}^{\prime} \in R^{\prime}$ for some $\varepsilon^{\prime} \leq \lambda^{-1}\left(\varepsilon-d_{\text {Spec }}\left(k_{1}^{\prime}, k_{2}\right)\right)$. Write $k_{1}^{\prime}=\left(a_{1}^{\prime}, x_{1}^{\prime}\right)$ and $k_{2}=\left(a_{2},\left[x_{2}, y_{2}\right]\right)$, define $x_{2}^{\prime}$

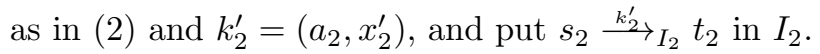

We show that the identity relation $\operatorname{id}_{S_{2}}=\left\{\left(s_{2}, s_{2}\right) \mid s_{2} \in S_{2}\right\} \subseteq S_{2} \times S_{2}$ witnesses $I_{2} \leq_{m} S_{2}$. Let first $s_{2} \stackrel{k_{2}^{\prime}}{\longrightarrow} I_{2} t_{2}$; we must have used one of the two constructions above for creating this transition. In the first case, we have $s_{2} \stackrel{-k_{2}}{-}{ }_{2}$ 
$t_{2}$ with $k_{2}^{\prime} \sqsubseteq k_{2}$, and in the second case, we have $s_{2} \stackrel{k_{2}}{\longrightarrow} 2 t_{2}$, hence also $s_{2} \stackrel{k_{2}}{\longrightarrow} \rightarrow_{2} t_{2}$, with the same property. For a transition $s_{2} \stackrel{k_{2}}{\longrightarrow} t_{2} t_{2}$ on the other hand, we have introduced $s_{2} \stackrel{k_{2}^{\prime}}{I_{2}} I_{2}$ in the second construction above, with $k_{2}^{\prime} \sqsubseteq k_{2}$.

We also want to show that the family $R^{\prime}$ is a witness for $d\left(I_{1}, I_{2}\right) \leq d_{m}\left(S_{1}, S_{2}\right)$. We have $\left(i_{1}^{0}, s_{2}^{0}\right) \in R_{d_{m}\left(S_{1}, S_{2}\right)}^{\prime}=R_{1} \circ R_{d_{m}\left(S_{1}, S_{2}\right)}$, so let $\left(i_{1}, s_{2}\right) \in R_{\varepsilon}^{\prime} \in R^{\prime}$ for some $\varepsilon \geq 0$. For any $i_{1} \stackrel{k_{1}^{\prime}}{\longrightarrow} I_{1} j_{1}$ we have $s_{2} \stackrel{-k_{2}}{\rightarrow} t_{2} t_{2}$ and $s_{2} \stackrel{k_{2}^{\prime}}{\stackrel{2}{2}_{I_{2}}} t_{2}$ by the first part of our construction above, with $d_{\text {Spec }}\left(k_{1}^{\prime}, k_{2}^{\prime}\right)=d_{\text {Spec }}\left(k_{1}^{\prime}, k_{2}\right) \leq \varepsilon$ because of (3), and also $\left(j_{1}, t_{2}\right) \in R_{\varepsilon^{\prime}}^{\prime} \in R^{\prime}$ for some $\varepsilon^{\prime} \leq \lambda^{-1}\left(\varepsilon-d_{\text {spec }}\left(k_{1}^{\prime}, k_{2}\right)\right)$. For any $s_{2} \stackrel{k_{2}^{\prime}}{\longrightarrow} I_{2} t_{2}$, we must have used one of the constructions above to introduce this transition, and both give us $i_{1} \stackrel{k_{1}^{\prime}}{\longrightarrow} I_{1} j_{1}$ with $d_{\text {Spec }}\left(k_{1}^{\prime}, k_{2}^{\prime}\right) \leq \varepsilon$ and $\left(j_{1}, t_{2}\right) \in R_{\varepsilon^{\prime}}^{\prime} \in R^{\prime}$ for some $\varepsilon^{\prime} \leq \lambda^{-1}\left(\varepsilon-d_{\text {spec }}\left(k_{1}^{\prime}, k_{2}\right)\right)$.

Theorem A.2. For WMTS $S_{1}, S_{2}$ with $S_{1}$ locally consistent and $S_{2}$ deterministic, we have $d_{t}\left(S_{1}, S_{2}\right)=d_{m}\left(S_{1}, S_{2}\right)$.

Proof. If $d_{t}\left(S_{1}, S_{2}\right)=\infty$, we are done by Theorem 1. Otherwise, let $R=\left\{R_{\varepsilon} \mid\right.$ $\varepsilon \geq 0\}$ be the smallest relation family for which

$$
\begin{aligned}
& -\left(s_{1}^{0}, s_{2}^{0}\right) \in R_{d_{t}\left(S_{1}, S_{2}\right)} \text { and } \\
& \text { - whenever }\left(s_{1}, s_{2}\right) \in R_{\varepsilon} \in R, s_{1} \stackrel{a, I_{\mathfrak{F}_{1}}}{\underline{\rightarrow}_{1}} t_{1}, s_{2} \stackrel{a, I_{3_{2}}}{ } t_{2} \text {, then } \\
& \quad\left(t_{1}, t_{2}\right) \in R_{\lambda^{-1}}\left(\varepsilon-d_{\text {Spec }}\left(\left(a, I_{1}\right),\left(a, I_{2}\right)\right)\right) .
\end{aligned}
$$

We show below that this definition makes sense (especially that $\varepsilon-d_{\text {Spec }}\left(\left(a, I_{1}\right),\left(a, I_{2}\right)\right) \geq 0$ in all cases $)$, and that $R$ is a modal refinement family. We will use the convenient notation $\left(s_{1}, S_{1}\right)$ for the WMTS $S_{1}$ with initial state $s_{1}^{0}$ replaced by $s_{1}$, similarly for $\left(s_{2}, S_{2}\right)$.

We first show inductively that for any pair of states $\left(s_{1}, s_{2}\right) \in R_{\varepsilon} \in R$ we have $d_{t}\left(\left(s_{1}, S_{1}\right),\left(s_{2}, S_{2}\right)\right) \leq \varepsilon$. This is obviously the case for $s_{1}=s_{1}^{0}$ and $s_{1}=s_{2}^{0}$, so assume now that $\left(s_{1}, s_{2}\right) \in R_{\varepsilon} \in R$ is such that $d_{t}\left(\left(s_{1}, S_{1}\right),\left(s_{2}, S_{2}\right)\right) \leq \varepsilon$ and let $s_{1} \stackrel{a, I_{3}}{\rightarrow_{1}} t_{1}, s_{2} \stackrel{a, I_{3}}{\underline{-}_{2}} t_{2}$. Let $\left(q_{1}^{\prime}, P_{1}^{\prime}\right) \in \llbracket\left(t_{1}, S_{1}\right) \rrbracket$ and $x_{1} \in I_{1}$.

There is an implementation $\left(p_{1}, P_{1}\right) \in \llbracket\left(s_{1}, S_{1}\right) \rrbracket$ for which $p_{1} \stackrel{a, x_{3}}{\longrightarrow} q_{1}$ and $\left(q_{1}, P_{1}\right) \leq_{m}\left(q_{1}^{\prime}, P_{1}^{\prime}\right)$. Now

$$
d_{t}\left(\left(p_{1}, P_{1}\right),\left(s_{2}, S_{2}\right)\right) \leq d_{t}\left(\left(p_{1}, P_{1}\right),\left(s_{1}, S_{1}\right)\right)+d_{t}\left(\left(s_{1}, S_{1}\right),\left(s_{2}, S_{2}\right)\right) \leq \varepsilon,
$$

hence we must have $s_{2} \stackrel{a_{2}^{\prime}, I_{2}^{\prime}}{\stackrel{I_{2}}{\prime}} t_{2}^{\prime}$ with $d_{\text {Spec }}\left(\left(a, x_{1}\right),\left(a_{2}^{\prime}, I_{2}^{\prime}\right)\right) \leq \varepsilon$. But then $a_{2}^{\prime}=a$, hence by determinism of $S_{2}, I_{2}=I_{2}^{\prime}$ and $t_{2}=t_{2}^{\prime}$.

The above considerations hold for any $x_{1} \in I_{1}$, hence $d_{\mathrm{Spec}}\left(\left(a, I_{1}\right),\left(a, I_{2}\right)\right) \leq$ $\varepsilon$. Thus $\varepsilon-d_{\mathrm{spec}}\left(\left(a, I_{1}\right),\left(a, I_{2}\right)\right) \geq 0$, and the definition of $R$ above is justified. Now let $x_{2} \in I_{2}$ such that $d_{\text {Spec }}\left(\left(a, x_{1}\right),\left(a, x_{2}\right)\right)=d_{\text {Spec }}\left(\left(a, x_{1}\right),\left(a, I_{2}\right)\right)$, then there is an implementation $\left(p_{2}, P_{2}\right) \in \llbracket\left(s_{2}, S_{2}\right) \rrbracket$ for which $p_{2} \stackrel{a, x_{2}}{\longrightarrow} q_{2}$, and

$$
\begin{aligned}
d\left(\left(q_{1}^{\prime}, P_{1}^{\prime}\right),\left(q_{2}, P_{2}\right)\right) & \leq \lambda^{-1}\left(\varepsilon-d_{\text {Spec }}\left(\left(a, x_{1}\right),\left(a, x_{2}\right)\right)\right) \\
& =\lambda^{-1}\left(\varepsilon-d_{\text {Spec }}\left(\left(a, I_{1}\right),\left(a, I_{2}\right)\right)\right),
\end{aligned}
$$

which, as $\left(q_{1}^{\prime}, P_{1}^{\prime}\right) \in \llbracket\left(t_{1}, S_{1}\right) \rrbracket$ was chosen arbitrarily, entails $d_{t}\left(\left(s_{1}, S_{1}\right),\left(s_{2}, S_{2}\right)\right) \leq$ $\lambda^{-1}\left(\varepsilon-d_{\mathrm{Spec}}\left(\left(a, I_{1}\right),\left(a, I_{2}\right)\right)\right)$. 
We are ready to show that $R$ is a refinement family. Let $\left(s_{1}, s_{2}\right) \in R_{\varepsilon} \in R$ for some $\varepsilon$, and assume $s_{1} \stackrel{k_{1}}{\rightarrow} 11 t_{1}$; by local consistency of $S$ we must have $k_{1} \neq \perp$, hence $k_{1}=\left(a, I_{1}\right)$. Let $x \in I_{1}$, then there is an implementation $\left(p, P^{x}\right) \in$ $\llbracket\left(s_{1}, S_{1}\right) \rrbracket$ with a transition $p \stackrel{m}{\longrightarrow} q$. Now $d_{t}\left(\left(p, P^{x}\right),\left(s_{2}, S_{2}\right)\right) \leq \varepsilon$, hence we have a transition $s_{2} \stackrel{a, I_{2}^{x}}{\rightarrow} t_{2}^{x}$ with $d_{\text {Spec }}\left((a, x),\left(a, I_{2}^{x}\right)\right) \leq \varepsilon$. Also for any other $x^{\prime} \in I_{1}$ we have a transition $s_{2} \stackrel{a, I_{2}^{x^{\prime}}}{\stackrel{x_{2}}{\rightarrow}} t_{2}^{x^{\prime}}$ with $d_{\text {Spec }}\left(\left(a, x^{\prime}\right),\left(a, I_{2}^{x^{\prime}}\right)\right) \leq \varepsilon$, hence by determinism of $S_{2}, I_{2}^{x}=I_{2}^{x^{\prime}}$ and $t_{2}^{x}=t_{2}^{x^{\prime}}$. It follows that there is a unique transition $s_{2} \stackrel{a, I_{3}}{\rightarrow} t_{2}$, and as $d_{\text {Spec }}\left((a, x),\left(a, I_{2}\right)\right) \leq \varepsilon$ for all $x \in I_{1}$, we have $d_{\text {Spec }}\left(\left(a, I_{1}\right),\left(a, I_{2}\right)\right) \leq \varepsilon$, and $\left(t_{1}, t_{2}\right) \in R_{\lambda^{-1}\left(\varepsilon-d_{\text {spec }}\left(\left(a, I_{1}\right),\left(a, I_{2}\right)\right)\right)}$ by definition.

Now assume $s_{2} \stackrel{k_{2}}{\longrightarrow} t_{2}$. If $k_{2}=\perp$, then there is a transition $s_{1} \stackrel{\perp}{\longrightarrow} t_{1}$ in $S_{1}$, in contradiction to local consistency of $S_{1}$. Hence $k_{2}=\left(a, I_{2}\right)$. Let $\left(p_{1}, P_{1}\right) \in$ $\llbracket\left(s_{1}, S_{1}\right) \rrbracket$, then we have $\left(p_{2}, P_{2}\right) \in \llbracket\left(s_{2}, S_{2}\right) \rrbracket$ with $d\left(\left(p_{1}, P_{1}\right),\left(p_{2}, P_{2}\right)\right) \leq \varepsilon$. Now any $\left(p_{2}, P_{2}\right) \in \llbracket\left(s_{2}, S_{2}\right) \rrbracket$ has $p_{2} \stackrel{a, x_{2}}{\longrightarrow} q_{2}$ with $x_{2} \in I_{2}$, thus there is also $p_{1} \stackrel{a, x_{1}}{\longrightarrow} q_{1}$ with $d_{\text {Spec }}\left(\left(a, x_{1}\right),\left(a, x_{2}\right)\right) \leq \varepsilon$ and $d\left(\left(q_{1}, P_{1}\right),\left(q_{2}, P_{2}\right)\right) \leq \lambda^{-1}(\varepsilon-$ $\left.d_{\text {Spec }}\left(\left(a, x_{1}\right),\left(a, x_{2}\right)\right)\right)$. This in turn implies that $s_{1} \stackrel{a, I_{1}}{\rightarrow} t_{1}$ for some $x_{1} \in I_{1}$. We will be done once we can show $d_{\text {Spec }}\left(\left(a, I_{1}\right),\left(a, I_{2}\right)\right) \leq \varepsilon$, so assume to the contrary that there is $x_{1}^{\prime} \in I_{1}$ with $d_{\text {Spec }}\left(\left(a, x_{1}\right),\left(a, I_{2}\right)\right)>\varepsilon$. Then there is an implementation $\left(p_{1}^{\prime}, P_{1}^{\prime}\right) \in \llbracket\left(s_{1}, S_{1}\right) \rrbracket$ with $p_{1}^{\prime} \stackrel{a, x_{1}^{\prime}}{\longrightarrow} q_{1}^{\prime}$, hence a transition $s_{2} \stackrel{a, I_{3}^{\prime}}{2} t_{2}^{\prime}$ with $d_{\text {Spec }}\left(\left(a, x_{1}^{\prime}\right),\left(a, I_{2}^{\prime}\right)\right) \leq \varepsilon$. But $I_{2}^{\prime}=I_{2}$ by determinism of $S_{2}$, a contradiction.

\section{Complexity Bounds}

The fact that computing thorough refinement distance is EXPTIME-hard is easy. By [4], deciding thorough refinement for MTS (without weights) is EXPTIMEcomplete. By translating MTS to WMTS with weight 0 on all transitions, deciding thorough refinement for modal transition systems polynomial-time reduces to deciding whether thorough refinement distance is $\leq 0$.

To show an upper bound on the complexity of computing modal refinement distance, we need to introduce discounted values of weighted games, cf. [19]. A weighted game graph is a finite real-weighted bipartite digraph $\left(V_{1}, V_{2}, \longrightarrow\right)$, i.e. with $V_{1} \cap V_{2}=\emptyset$ and $\longrightarrow \in\left(V_{1} \times \mathbb{R} \times V_{2}\right) \cup\left(V_{2} \times \mathbb{R} \times V_{1}\right)$ a finite set of edges. These are assumed to be non-blocking in the sense that each $v \in V_{1} \cup V_{2}$ has at least one outgoing edge $v \stackrel{r}{\longrightarrow} w$ (which is the shorthand for $(v, r, w) \in \longrightarrow$ ).

A Player-1 strategy in such a weighted game graph is a mapping $\theta_{1}: V_{1} \rightarrow$ $\mathbb{R} \times V_{2}$ for which $\left(v_{1}, \theta_{1}\left(v_{1}\right)\right) \in \longrightarrow$ for each $v_{1} \in V_{1}$. Similarly, a Player-2 strategy is a mapping $\theta_{2}: V_{2} \rightarrow \mathbb{R} \times V_{1}$ such that $\left(v_{2}, \theta_{2}\left(v_{2}\right)\right) \in \longrightarrow$ for each $v_{2} \in V_{2}$. The sets of all Player- 1 and Player-2 strategies are denoted $\Theta_{1}$ and $\Theta_{2}$, respectively.

Denote by $\operatorname{tgt}(e)=w$ the target of an edge $e=(v, r, w) \in \longrightarrow$ and by $w t(e)=r$ its weight. A vertex $v_{0} \in V_{1}$ and a pair $\left(\theta_{1}, \theta_{2}\right) \in \Theta_{1} \times \Theta_{2}$ of strategies determine a unique infinite sequence $\left(e_{j}\left(\theta_{1}, \theta_{2}\right)\right)_{j \geq 0}$ of edges $e_{j}\left(\theta_{1}, \theta_{2}\right) \in \longrightarrow$ for 
which

$$
\begin{aligned}
e_{0}\left(\theta_{1}, \theta_{2}\right) & =\left(v_{0}, \theta_{1}\left(v_{0}\right)\right), \\
e_{2 j+1}\left(\theta_{1}, \theta_{2}\right) & =\left(\operatorname{tgt}\left(e_{2 j}\right), \theta_{2}\left(\operatorname{tgt}\left(e_{2 j}\right)\right)\right), \\
e_{2 j}\left(\theta_{1}, \theta_{2}\right) & =\left(\operatorname{tgt}\left(e_{2 j-1}, \theta_{1}\left(\operatorname{tgt}\left(e_{2 j-1}\right)\right)\right) .\right.
\end{aligned}
$$

In other words, the two players alternate to pick edges in $\longrightarrow$ according to their strategies. The discounted value of the game $\left(V_{1}, V_{2}, \longrightarrow\right)$ played from $v_{0} \in V_{1}$ with discounting factor $\lambda, 0 \leq \lambda<1$, is defined to be

$$
p\left(v_{0}, \lambda\right)=\sup _{\theta_{1} \in \Theta_{1}} \inf _{\theta_{2} \in \Theta_{2}} \sum_{j=0}^{\infty} \lambda^{j} w t\left(e_{j}\left(\theta_{1}, \theta_{2}\right)\right) .
$$

We recall the following theorem from [19]; the complexity result is obtained by reduction to simple stochastic games [7].

Lemma 1 ([19]). The discounted value $p\left(v_{0}, \lambda\right)$ may be computed as the unique fixed point to the equations

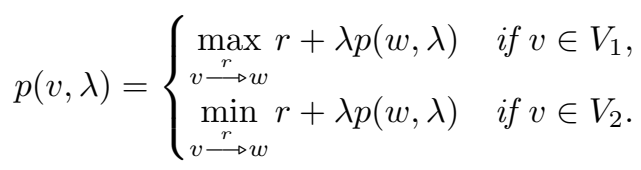

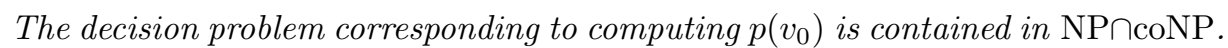

Next we present a reduction from modal refinement distance of WMTS to discounted values of weighted games, $c f$. . [11].

Lemma 2. For WMTS $S_{1}, S_{2}$ one can construct in polynomial time a weighted game $\left(V_{1}, V_{2}, \longrightarrow\right)$ with a vertex $v_{0} \in V_{1}$ such that $d_{m}\left(S_{1}, S_{2}\right)=p\left(v_{0}, \sqrt{\lambda}\right)$.

Proof. Let $V_{1}=S_{1} \times S_{2}, V_{2}=S_{1} \times S_{2} \times$ Spec $\times\{$ may, must $\}$, and define the transitions as follows:

$$
\begin{array}{rlr}
\left(s_{1}, s_{2}\right) \stackrel{0}{\longrightarrow}\left(t_{1}, s_{2}, k_{1}, \text { may }\right) & \text { if } & s_{1} \stackrel{\underline{k}_{1}}{\rightarrow} t_{1} \\
\left(s_{1}, s_{2}\right) \stackrel{0}{\longrightarrow}\left(s_{1}, t_{2}, k_{2}, \text { must }\right) & \text { if } & s_{2} \stackrel{k_{2}}{\longrightarrow} t_{2} \\
\left(t_{1}, s_{2}, k_{1}, \text { may }\right) \stackrel{d_{\text {spec }}\left(k_{1}, k_{2}\right)}{\longrightarrow}\left(t_{1}, t_{2}\right) & \text { if } & s_{2} \stackrel{\underline{k}_{2} \rightarrow_{2}}{\longrightarrow} t_{2} \\
\left(s_{1}, t_{2}, k_{2}, \text { must }\right) \stackrel{d_{\text {spec }}\left(k_{1}, k_{2}\right)}{\longrightarrow}\left(t_{1}, t_{2}\right) & \text { if } & s_{1} \stackrel{k_{1}}{\longrightarrow} t_{1}
\end{array}
$$

Setting $v_{0}=\left(s_{1}^{0}, s_{2}^{0}\right)$ finishes the construction.

In [11] it is also shown that conversely, computing discounted values of weighted games may be polynomial-time reduced to computing simulation distance for weighted transition systems, hence we can conclude the following.

Lemma 3. The decision problem corresponding to computing modal refinement distance for WMTS is polynomial-time equivalent to the decision problem corresponding to computing discounted values of weighted games. 


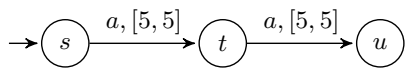

(a) $S$

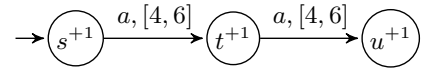

(b) $S^{+1}$

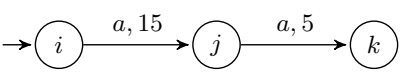

(c) $I$

Fig. 4. WMTS $S$ for which $\llbracket S^{\prime} \rrbracket \subsetneq \llbracket S \rrbracket^{+\varepsilon}$.

\section{Appendix: Proofs for Section 4}

Proof (of Proposition 1). By the triangle inequality we have

$$
\begin{aligned}
& d_{m}\left(S_{1}, S_{2}^{\prime}\right) \leq d_{m}\left(S_{1}, S_{2}\right)+d_{m}\left(S_{2}, S_{2}^{\prime}\right), \\
& d_{m}\left(S_{1}, S_{2}\right) \leq d_{m}\left(S_{1}, S_{2}^{\prime}\right)+d_{m}\left(S_{2}^{\prime}, S_{2}\right), \\
& d_{m}\left(S_{1}, S_{2}\right) \leq d_{m}\left(S_{1}, S_{1}^{\prime}\right)+d_{m}\left(S_{1}^{\prime}, S_{2}\right), \\
& d_{m}\left(S_{1}^{\prime}, S_{2}\right) \leq d_{m}\left(S_{1}^{\prime}, S_{1}\right)+d_{m}\left(S_{1}, S_{2}\right) .
\end{aligned}
$$

Proof (of Proposition 2). For the first claim, the identity relation $\operatorname{id}_{S}=\{(s, s) \mid$ $s \in S\} \subseteq S \times S$ is a witness for $S \leq_{m} S^{+\delta}$ : if $s \stackrel{-k}{\rightarrow} t$, then by construction $s \stackrel{k_{2}}{\longrightarrow}+\delta t$ with $k \sqsubseteq k_{2}$, and if $s \stackrel{k_{2}}{\longrightarrow}+\delta t$, then again by construction $s \stackrel{k}{\longrightarrow} t$ for some $k \sqsubseteq k_{2}$.

Now to prove $d_{m}\left(S^{+\delta}, S\right) \leq(1-\lambda)^{-1} \delta$, we define a family of relations $R=$ $\left\{R_{\varepsilon} \mid \varepsilon \geq 0\right\}$ by $R_{\varepsilon}=\emptyset$ for $\varepsilon<(1-\lambda)^{-1} \delta$ and $R_{\varepsilon}=\operatorname{id}_{S}$ for $\varepsilon \geq(1-\lambda)^{-1} \delta$. We show that $R$ is a modal refinement family.

Let $(s, s) \in R_{\varepsilon}$ for some $\varepsilon \geq(1-\lambda)^{-1} \delta$, and assume $s \stackrel{{ }^{k_{2}} \rightarrow}{\rightarrow}+\delta$. By construction there is a transition $s \stackrel{-k}{\rightarrow} t$ with $d_{\text {Spec }}\left(k_{2}, k\right) \leq \delta \leq \varepsilon$. Now

$$
\frac{1}{\lambda}\left(\varepsilon-d_{\mathrm{Spec}}\left(k_{2}, k\right)\right) \geq \frac{1}{\lambda}\left(\frac{\delta}{1-\lambda}-\delta\right)=\frac{\delta}{1-\lambda} \geq \varepsilon
$$

and $(t, t) \in R_{\varepsilon}$, which settles this part of the proof. The other direction, starting with a transition $s \stackrel{k}{\longrightarrow} t$, is similar.

Proof (of Proposition 3). If $I \in \llbracket S^{\prime} \rrbracket$, then $d_{m}\left(I, S^{\prime}\right)=0$, hence $d_{m}(I, S) \leq \varepsilon$ by Proposition 1, which in turn implies that $I \in \llbracket S \rrbracket^{+\varepsilon}$. The example in Figure 4 then shows that in general, $\llbracket S^{\prime} \rrbracket \subsetneq \llbracket S \rrbracket^{+\varepsilon}$. For $\delta=1$ and $\lambda=.9$, we have $I \in \llbracket S \rrbracket^{+(1-\lambda)^{-1} \delta}$, but $I \notin \llbracket S^{+\delta} \rrbracket$.

\section{Appendix: Proofs for Section 5}

Theorem A.3. There is no unary operator $\mathcal{D}$ on WMTS for which it holds that (3.1) $\mathcal{D}(S)$ is deterministic for any WMTS $S$, 


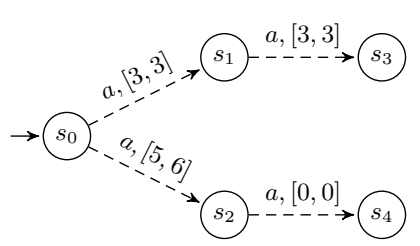

(a) $S$

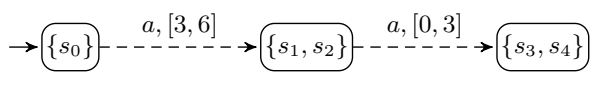

(b) $\mathcal{D}^{\prime}(S)$

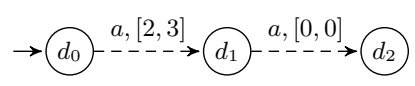

(c) $D$

Fig. 5. WMTS for which $d_{m}\left(\mathcal{D}^{\prime}(S), D\right) \not \leq d_{m}(S, D)$.

(3.2) $S \leq_{m} \mathcal{D}(S)$ for any WMTS $S$,

(3.3) $S \leq_{m}^{\varepsilon} D$ implies $\mathcal{D}(S) \leq_{m}^{\varepsilon} D$ for any WMTS $S$, any deterministic WMTS $D$, and any $\varepsilon \geq 0$.

Proof. There is a determinization operator $\mathcal{D}^{\prime}$ on WMTS, introduced in [3], which satisfies Property (3.2) above and a weaker version of Property (3.3) with $\varepsilon=0$ :

$\left(3.3^{\prime}\right) S \leq_{m} D$ implies $\mathcal{D}^{\prime}(S) \leq_{m} D$ for any WMTS $S$ and any deterministic WMTS $D$.

Assume now that there is an operator $\mathcal{D}$ as in the theorem. Then for any WMTS $S, S \leq_{m} \mathcal{D}^{\prime}(S)$ and thus $\mathcal{D}(S) \leq_{m} \mathcal{D}^{\prime}(S)$ by $(3.3)$, and $S \leq_{m} \mathcal{D}(S)$ and hence $\mathcal{D}^{\prime}(S) \leq_{m} \mathcal{D}(S)$ by $\left(3.3^{\prime}\right)$. We finish the proof by showing that the operator $\mathcal{D}^{\prime}$ from [3] does not satisfy (3.3). The example in Figure 5 shows a WMTS $S$ and a deterministic WMTS $D$ for which $d_{m}\left(\mathcal{D}^{\prime}(S), D\right)=3+3 \lambda$ and $d_{m}(S, D)=$ $\max (3,3 \lambda)=3$, hence $d_{m}\left(\mathcal{D}^{\prime}(S), D\right) \not \leq d_{m}(S, D)$.

Theorem A.4. There is no partial binary operator $\wedge$ on WMTS for which it holds that

(4.1) $S_{1} \wedge S_{2} \leq_{m} S_{1}$ and $S_{1} \wedge S_{2} \leq_{m} S_{2}$ for all locally consistent WMTS $S_{1}, S_{2}$ for which $S_{1} \wedge S_{2}$ is defined,

(4.2) for any locally consistent WMTS $S$ and all deterministic and locally consistent WMTS $S_{1}, S_{2}$ such that $S \leq_{m} S_{1}$ and $S \leq_{m} S_{1}, S_{1} \wedge S_{2}$ is defined and $S \leq_{m} S_{1} \wedge S_{2}$,

(4.3) for any $\varepsilon \geq 0$, there exist $\varepsilon_{1} \geq 0$ and $\varepsilon_{2} \geq 0$ such that for any locally consistent WMTS $S$ and all deterministic and locally consistent WMTS $S_{1}$, $S_{2}$ for which $S_{1} \wedge S_{2}$ is defined, $S \leq_{m}^{\varepsilon_{1}} S_{1}$ and $S \leq_{m}^{\varepsilon_{2}} S_{2}$ imply $S \leq_{m}^{\varepsilon} S_{1} \wedge S_{2}$.

Proof. We follow the same strategy as in the proof of Theorem 3. There is a conjunction operator $\wedge^{\prime}$ defined for WMTS in [3] which satisfies Properties (4.1) 


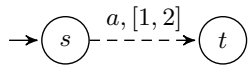

(a) $S$

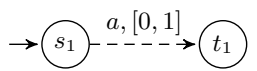

(b) $S_{1}$

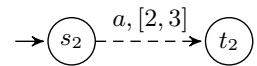

(c) $S_{2}$

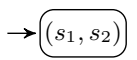

(d) $S_{1} \wedge S_{2}$

Fig. 6. WMTS for which $d_{m}\left(S, S_{1} \wedge S_{2}\right) \not \leq \max \left(d_{m}\left(S, S_{1}\right), d_{m}\left(S, S_{2}\right)\right)$.

and (4.2) and a version (4.3') for $\varepsilon=0$ of Property (4.3). Using these properties, one can see that for all deterministic and locally consistent WMTS $S_{1}$ and $S_{2}$ for which $S_{1} \wedge S_{2}$ and $S_{1} \wedge^{\prime} S_{2}$ are locally consistent, $S_{1} \wedge S_{2} \leq_{m} S_{1} \wedge^{\prime} S_{2}$ and $S_{1} \wedge^{\prime} S_{2} \leq_{m} S_{1} \wedge S_{2}$. The WMTS depicted in Figure 6 then show that $d_{m}\left(S, S_{1} \wedge S_{2}\right) \leq \max \left(d_{m}\left(S, S_{1}\right), d_{m}\left(S, S_{2}\right)\right)$ does not hold in general. Indeed, $d_{m}\left(S, S_{1}\right)=d_{m}\left(S, S_{2}\right)=1$, but $d_{m}\left(S, S_{1} \wedge S_{2}\right)=\infty$.

\section{Appendix: Proofs for Section 6}

Lemma 4. For $k_{1}, k_{2}, k_{3}, k_{4} \in$ Spec with $k_{1} \oplus k_{3}$ and $k_{2} \oplus k_{4}$ defined, we have $d_{\text {Spec }}\left(k_{1} \oplus k_{3}, k_{2} \oplus k_{4}\right) \leq d_{\text {Spec }}\left(k_{1}, k_{2}\right)+d_{\text {Spec }}\left(k_{3}, k_{4}\right)$.

Proof (of Lemma 4). If $k_{1}=\perp$ or $k_{3}=\perp$, then the left-hand side of the inequality is 0 , and if $k_{2}=\perp$ or $k_{4}=\perp$, then the right-hand side is $\infty$, hence in both cases the proof is complete. We are left with the case where all $k_{i} \neq \perp$; write $k_{i}=\left(a,\left[x_{i}, y_{i}\right]\right)$ for all $i$. We have

$$
\begin{aligned}
d_{\text {Spec }}\left(k_{1} \oplus k_{3}, k_{2} \oplus k_{4}\right) & =\max \left(\left(x_{2}+x_{4}\right) \dot{-}\left(x_{1}+x_{3}\right),\left(y_{1}+y_{3}\right) \dot{-}\left(y_{2}+y_{4}\right)\right), \\
d_{\text {Spec }}\left(k_{1}, k_{2}\right)+d_{\text {Spec }}\left(k_{3}, k_{4}\right) & =\max \left(x_{2} \dot{-} x_{1}, y_{1} \dot{-} y_{2}\right)+\max \left(x_{4} \dot{-} x_{3}, y_{3} \dot{-} y_{4}\right) \\
& \geq \max \left(\left(x_{2} \dot{-} x_{1}\right)+\left(x_{4} \dot{-} x_{3}\right),\left(y_{1} \dot{-} y_{2}\right)+\left(y_{3} \dot{-} y_{4}\right)\right) .
\end{aligned}
$$

But

$$
\begin{aligned}
\left(x_{2}+x_{4}\right) \doteq\left(x_{1}+x_{3}\right) & =\max \left(\left(x_{2}+x_{4}\right)-\left(x_{1}+x_{3}\right), 0\right) \\
& =\max \left(\left(x_{2}-x_{1}\right)+\left(x_{4}-x_{3}\right), 0\right) \\
& \leq \max \left(x_{2}-x_{1}, 0\right)+\max \left(x_{4}-x_{3}, 0\right) \\
& =\left(x_{2} \dot{-} x_{1}\right)+\left(x_{4} \dot{-} x_{3}\right),
\end{aligned}
$$

and similarly one shows $\left(y_{1}+y_{3}\right) \doteq\left(y_{2}+y_{4}\right) \leq\left(y_{1} \dot{-} y_{2}\right)+\left(y_{3} \dot{-} y_{4}\right)$.

Proof (of Theorem 5). If $d_{m}\left(S_{1}, S_{2}\right)=\infty$ or $d_{m}\left(S_{3}, S_{4}\right)=\infty$, we have nothing to prove. Otherwise, let $R^{1}=\left\{R_{\varepsilon}^{1} \subseteq S_{1} \times S_{2} \mid \varepsilon \geq 0\right\}, R^{2}=\left\{R_{\varepsilon}^{2} \subseteq S_{3} \times\right.$ $\left.S_{4} \mid \varepsilon \geq 0\right\}$ be witnesses for $d_{m}\left(S_{1}, S_{2}\right)$ and $d_{m}\left(S_{3}, S_{4}\right)$, respectively; hence $\left(s_{1}^{0}, s_{2}^{0}\right) \in R_{d_{m}\left(S_{1}, S_{2}\right)}^{1} \in R^{1}$ and $\left(s_{3}^{0}, s_{4}^{0}\right) \in R_{d_{m}\left(S_{3}, S_{4}\right)}^{2} \in R^{2}$. Define

$$
\begin{aligned}
R_{\varepsilon}=\left\{\left(\left(s_{1}, s_{3}\right),\left(s_{2}, s_{4}\right)\right)\right. & \in S_{1} \times S_{3} \times S_{2} \times S_{4} \mid \\
\left(s_{1}, s_{2}\right) & \left.\in R_{\varepsilon_{1}}^{1} \in R^{1},\left(s_{3}, s_{4}\right) \in R_{\varepsilon_{2}}^{2} \in R^{2}, \varepsilon_{1}+\varepsilon_{2} \leq \varepsilon\right\}
\end{aligned}
$$


for all $\varepsilon \geq 0$ and let $R=\left\{R_{\varepsilon} \mid \varepsilon \geq 0\right\}$. We show that $R$ is a witness for $d_{m}\left(S_{1}\left\|S_{3}, S_{2}\right\| S_{4}\right) \leq d_{m}\left(S_{1}, S_{2}\right)+d_{m}\left(S_{3}, S_{4}\right)$.

We have $\left(\left(s_{1}^{0}, s_{3}^{0}\right),\left(s_{2}^{0}, s_{4}^{0}\right)\right) \in R_{d_{m}\left(S_{1}, S_{2}\right)+d_{m}\left(S_{3}, S_{4}\right)} \in R$. Now let $\left(\left(s_{1}, s_{3}\right),\left(s_{2}, s_{4}\right)\right) \in$ $R_{\varepsilon} \in R$ for some $\varepsilon$, then $\left(s_{1}, s_{2}\right) \in R_{\varepsilon_{1}}^{1} \in R^{1}$ and $\left(s_{3}, s_{4}\right) \in R_{\varepsilon_{2}}^{2} \in R^{2}$ for some $\varepsilon_{1}+\varepsilon_{2} \leq \varepsilon$.

Assume $\left(s_{1}, s_{3}\right) \stackrel{k_{1} \underset{\oplus}{\rightarrow}}{\rightarrow}\left(t_{1}, t_{3}\right)$, then $s_{1} \stackrel{k_{1}}{\rightarrow}{ }_{1} t_{1}$ and $s_{3} \stackrel{k_{3}}{\rightarrow} 3 t_{3}$. By $\left(s_{1}, s_{2}\right) \in$ $R_{\varepsilon_{1}}^{1} \in R^{1}$, we have $s_{2} \stackrel{{ }_{2} \rightarrow_{2}}{\rightarrow} t_{2}$ with $d_{\text {Spec }}\left(k_{1}, k_{2}\right) \leq \varepsilon_{1}$ and $\left(t_{1}, t_{2}\right) \in R_{\varepsilon_{1}^{\prime}}^{1} \in R^{1}$ for some $\varepsilon_{1}^{\prime} \leq \lambda^{-1}\left(\varepsilon_{1}-d_{\text {Spec }}\left(k_{1}, k_{2}\right)\right)$; similarly, $s_{4}{\stackrel{-{ }^{k}}{-} \rightarrow_{4}}_{4} t_{4}$ with $d_{\text {Spec }}\left(k_{3}, k_{4}\right) \leq \varepsilon_{2}$ and $\left(t_{3}, t_{4}\right) \in R_{\varepsilon_{2}^{\prime}}^{2} \in R^{2}$ for some $\varepsilon_{2}^{\prime} \leq \lambda^{-1}\left(\varepsilon_{2}-d_{\text {Spec }}\left(k_{3}, k_{4}\right)\right)$. Let $\varepsilon^{\prime}=\varepsilon_{1}^{\prime}+\varepsilon_{2}^{\prime}$, then the sum $k_{2} \oplus k_{4}$ is defined, and

$$
\begin{aligned}
\varepsilon^{\prime} & \leq \lambda^{-1}\left(\varepsilon_{1}+\varepsilon_{2}-\left(d_{\mathrm{Spec}}\left(k_{1}, k_{2}\right)+d_{\mathrm{Spec}}\left(k_{3}, k_{4}\right)\right)\right) \\
& \leq \lambda^{-1}\left(\varepsilon-d_{\mathrm{Spec}}\left(k_{1} \oplus k_{3}, k_{2} \oplus k_{4}\right)\right)
\end{aligned}
$$

by Lemma 4 . We have $\left(s_{2}, s_{4}\right) \stackrel{k_{2} \oplus \underline{k}_{4}}{\rightarrow}\left(t_{2}, t_{4}\right), d_{\text {Spec }}\left(k_{1} \oplus k_{3}, k_{2} \oplus k_{4}\right) \leq \varepsilon_{1}+\varepsilon_{2} \leq \varepsilon$ again by Lemma 4 , and $\left(\left(t_{1}, t_{3}\right),\left(t_{2}, t_{4}\right)\right) \in R_{\varepsilon^{\prime}} \in R$.

The reverse direction, starting with a transition $\left(s_{2}, s_{4}\right) \stackrel{k_{2} \oplus k_{4}}{\longrightarrow}\left(t_{2}, t_{4}\right)$, is similar.

Lemma 5. If $k_{1}, k_{2}, k_{3} \in \mathrm{Spec}$ are such that $k_{1} \ominus k_{2}$ and $k_{2} \oplus k_{3}$ are defined and $k_{1} \ominus k_{2} \neq \perp, k_{2} \oplus k_{3} \neq \perp$, then $d_{\text {Spec }}\left(k_{3}, k_{1} \ominus k_{2}\right)=d_{\text {Spec }}\left(k_{2} \oplus k_{3}, k_{1}\right)$.

Proof (of Lemma 5). We can write $k_{i}=\left(a,\left[x_{i}, y_{i}\right]\right)$ for some $a \in \Sigma$. Then

$$
\begin{aligned}
& d_{\text {Spec }}\left(k_{3}, k_{1} \ominus k_{2}\right)=\max \left(\left(x_{1}-x_{2}\right) \doteq x_{3}, y_{3} \dot{-}\left(y_{1}-y_{2}\right)\right)
\end{aligned}
$$

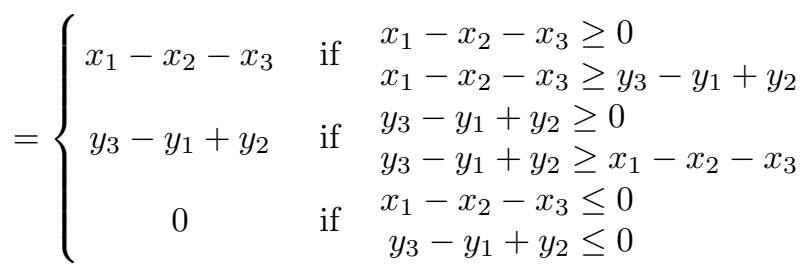

and similarly

$$
\begin{aligned}
d_{\text {Spec }}\left(k_{2} \oplus k_{3}, k_{1}\right)= & \max \left(x_{1} \dot{-}\left(x_{2}+x_{3}\right),\left(y_{2}+y_{3}\right) \dot{-} y_{1}\right) \\
= & \left\{\begin{array}{lll}
x_{1}-x_{2}-x_{3} & \text { if } \quad \begin{array}{l}
x_{1}-x_{2}-x_{3} \geq 0 \\
\end{array} & x_{1}-x_{2}-x_{3} \geq y_{2}+y_{3}-y_{1} \\
y_{2}+y_{3}-y_{1} & \text { if } \quad \begin{array}{l}
y_{2}+y_{3}-y_{1} \geq 0 \\
y_{2}+y_{3}-y_{1} \geq x_{1}-x_{2}-x_{3}
\end{array} \\
0 & \text { if } \begin{array}{l}
x_{1}-x_{2}-x_{3} \leq 0 \\
y_{2}+y_{3}-y_{1} \leq 0
\end{array}
\end{array}\right.
\end{aligned}
$$

Proof (of Theorem 6). To avoid confusion, we write $-\rightarrow \Downarrow$ and $\longrightarrow \Downarrow$ for transitions in $S_{1} \| S_{2}$ and $\rightarrow_{\|}$and $\longrightarrow \|$ for transitions in $S_{2} \| S_{3}$. The inequality $d_{m}\left(S_{3}, S_{1} \| S_{2}\right) \geq d_{m}\left(S_{2} \| S_{3}, S_{1}\right)$ is trivial if $d_{m}\left(S_{2} \| S_{3}, S_{1}\right)=\infty$, so assume the opposite and let $R^{1}=\left\{R_{\varepsilon}^{1} \subseteq S_{3} \times\left(S_{1} \times S_{2} \cup\{u\}\right) \mid \varepsilon \geq 0\right\}$ be 
a witness for $d_{m}\left(S_{3}, S_{1} \| S_{2}\right)$. Define $R_{\varepsilon}^{2}=\left\{\left(\left(s_{2}, s_{3}\right), s_{1}\right) \mid\left(s_{3},\left(s_{1}, s_{2}\right)\right) \in\right.$ $\left.R_{\varepsilon}^{1}\right\} \subseteq S_{2} \times S_{3} \times S_{1}$ for all $\varepsilon \geq 0$, and let $R^{2}=\left\{R_{\varepsilon}^{2} \mid \varepsilon \geq 0\right\}$. Certainly $\left(\left(s_{2}^{0}, s_{3}^{0}\right), s_{1}^{0}\right) \in R_{d_{m}\left(S_{3}, S_{1} \Downarrow S_{2}\right)}^{2} \in R^{2}$, so let now $\left(\left(s_{2}, s_{3}\right), s_{1}\right) \in R_{\varepsilon}^{2} \in R^{2}$ for some $\varepsilon \geq 0$.

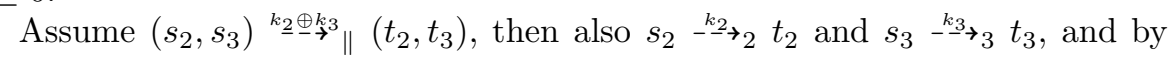

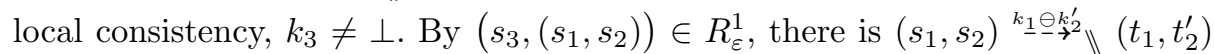
for which $d_{\text {Spec }}\left(k_{3}, k_{1} \ominus k_{2}^{\prime}\right)=d_{\text {spec }}\left(k_{2}^{\prime} \oplus k_{3}, k_{1}\right) \leq \varepsilon$ and such that $\left(t_{3},\left(t_{1}, t_{2}^{\prime}\right)\right) \in$ $R_{\varepsilon^{\prime}}^{1} \in R^{1}$, hence $\left(\left(t_{2}^{\prime}, t_{3}\right), t_{1}\right) \in R_{\varepsilon^{\prime}}^{2} \in R^{2}$, for some $\varepsilon^{\prime} \leq \lambda^{-1}\left(\varepsilon-d_{\text {Spec }}\left(k_{2}^{\prime} \oplus\right.\right.$ $\left.\left.k_{3}, k_{1}\right)\right)$. By definition of quotient we must have $s_{1} \stackrel{-k_{1}}{\rightarrow} t_{1}$ and $s_{2}-{\stackrel{-k}{k_{2}}}_{2} t_{2}^{\prime}$, and by determinism of $S_{2}, k_{2}^{\prime}=k_{2}$ and $t_{2}^{\prime}=t_{2}$.

Assume $s_{1} \stackrel{k_{1}}{\longrightarrow} S_{1} t_{1}$, then we have $k_{1} \neq \perp$ by local consistency of $S_{1}$. We must have a transition $s_{2} \stackrel{k_{2}}{\longrightarrow}{ }_{2} t_{2}$ for which $k_{1} \ominus k_{2}$ is defined and $k_{1} \ominus k_{2} \neq \perp$, for otherwise the state $\left(s_{1}, s_{2}\right)$ would have been pruned from $S_{1} \| S_{2}$. Hence $\left(s_{1}, s_{2}\right) \stackrel{k_{1} \ominus k_{2}}{\longrightarrow} \|\left(t_{1}, t_{2}\right)$. This in turn implies that there is $s_{3} \stackrel{k_{3}}{\longrightarrow}{ }_{3} t_{3}$ for which $d_{\text {Spec }}\left(k_{3}, k_{1} \ominus k_{2}\right)=d_{\text {Spec }}\left(k_{2} \oplus k_{3}, k_{1}\right) \leq \varepsilon$ and such that $\left(t_{3},\left(t_{1}, t_{2}\right)\right) \in R_{\varepsilon^{\prime}}^{1} \in R^{1}$, hence $\left(\left(t_{2}, t_{3}\right), t_{1}\right) \in R_{\varepsilon^{\prime}}^{2} \in R^{2}$, for some $\varepsilon^{\prime} \leq \lambda^{-1}\left(\varepsilon-d_{\text {Spec }}\left(k_{2} \oplus k_{3}, k_{1}\right)\right)$, and by definition of parallel composition, $\left(s_{2}, s_{3}\right) \stackrel{k_{2} \oplus k_{3}}{\longrightarrow} \|\left(t_{2}, t_{3}\right)$.

To show that $d_{m}\left(S_{3}, S_{1} \| S_{2}\right) \leq d_{m}\left(S_{2} \| S_{3}, S_{1}\right)$, let $R^{2}=\left\{R_{\varepsilon}^{2} \subseteq S_{2} \times\right.$ $\left.S_{3} \times S_{1} \mid \varepsilon \geq 0\right\}$ be a witness for $d_{m}\left(S_{2} \| S_{3}, S_{1}\right)$, define $R_{\varepsilon}^{1}=\left\{\left(s_{3},\left(s_{1}, s_{2}\right)\right) \mid\right.$ $\left.\left(\left(s_{2}, s_{3}\right), s_{1}\right) \in R_{\varepsilon}^{2}\right\} \cup\left\{\left(s_{3}, u\right) \mid s_{3} \in S_{3}\right\}$ for all $\varepsilon \geq 0$, and let $R^{1}=\left\{R_{\varepsilon}^{1} \mid \varepsilon \geq 0\right\}$, then $\left(s_{3}^{0},\left(s_{1}^{0}, s_{2}^{0}\right)\right) \in R_{d_{m}\left(S_{2} \| S_{3}, S_{1}\right)}^{1} \in R^{1}$.

For any $\left(s_{3}, u\right) \in R_{\varepsilon}^{1}$ for some $\varepsilon \geq 0$, any transition $s_{3} \stackrel{-k_{3}}{\rightarrow}{ }_{3} t_{3}$ can be matched by $u \stackrel{k_{3}}{\rightarrow} \| u$, and then $\left(t_{3}, u\right) \in R_{0}^{1}$. Let now $\left(s_{3},\left(s_{1}, s_{2}\right)\right) \in R_{\varepsilon}^{1}$ for some $\varepsilon \geq 0$, and assume $s_{3} \stackrel{k_{3}}{\rightarrow} t_{3}$. If $k_{2} \oplus k_{3}$ is undefined for all transitions $s_{2} \stackrel{-k_{2}}{\rightarrow} t_{2} t_{2}$, then by definition $\left(s_{1}, s_{2}\right) \stackrel{k_{3}}{\rightarrow} u$, and again $\left(t_{3}, u\right) \in R_{0}^{1}$. If there is a transition $s_{2} \stackrel{k_{2}}{-\stackrel{k_{2}}{\rightarrow}} t_{2}$ such that $k_{2} \oplus k_{3}$ is defined, then also $\left(s_{2}, s_{3}\right) \stackrel{{ }_{2}{ }_{2} \oplus_{3}}{\rightarrow} \|\left(t_{2}, t_{3}\right)$. Hence we have $s_{1} \stackrel{k_{1}}{\rightarrow} 11$ with $d_{\text {Spec }}\left(k_{2} \oplus k_{3}, k_{1}\right) \leq \varepsilon$, implying that $\left(s_{1}, s_{2}\right) \stackrel{k_{1} \ominus k_{2}}{\rightarrow} \|$ $\left(t_{1}, t_{2}\right)$. We must have $k_{1} \ominus k_{2} \neq \perp$, for otherwise $d_{\text {Spec }}\left(S_{3}, S_{1} \| S_{2}\right)=\infty$, hence $d_{\text {Spec }}\left(k_{3}, k_{1} \ominus k_{2}\right)=d_{\text {spec }}\left(k_{2} \oplus k_{3}, k_{1}\right) \leq \varepsilon$. Also, $\left(\left(t_{2}, t_{3}\right), t_{1}\right) \in R_{\varepsilon^{\prime}}^{2} \in R^{2}$, hence $\left(t_{3},\left(t_{1}, t_{2}\right)\right) \in R_{\varepsilon^{\prime}}^{1} \in R^{1}$, for some $\varepsilon^{\prime} \leq \lambda^{-1}\left(\varepsilon-d_{\text {spec }}\left(k_{3}, k_{1} \ominus k_{2}\right)\right)$.

Assume $\left(s_{1}, s_{2}\right) \stackrel{k_{1} \ominus k_{2}}{\longrightarrow} \|\left(t_{1}, t_{2}\right)$, then $k_{1} \ominus k_{2} \neq \perp$ by local consistency of $S_{1} \| S_{2}$, hence we have $s_{1} \stackrel{k_{1}}{\longrightarrow}{ }_{1} t_{1}$ and $s_{2} \stackrel{k_{2}}{\longrightarrow} t_{2} t_{2}$. It follows that $\left(s_{2}, s_{3}\right) \stackrel{k_{2}^{\prime} \oplus k_{3}}{\rightarrow} \|\left(t_{2}^{\prime}, t_{3}\right)$ with $d_{\text {Spec }}\left(k_{2}^{\prime} \oplus k_{3}, k_{1}\right)=d_{\text {Spec }}\left(k_{3}, k_{1} \ominus k_{2}^{\prime}\right) \leq \varepsilon$ and such that $\left(\left(t_{2}^{\prime}, t_{3}\right), t_{1}\right) \in R_{\varepsilon^{\prime}}^{2} \in$ $R^{2}$, hence $\left(t_{3},\left(t_{1}, t_{2}^{\prime}\right)\right) \in R_{\varepsilon^{\prime}}^{1} \in R^{1}$, for some $\varepsilon^{\prime} \leq \lambda^{-1}\left(\varepsilon-d_{\text {Spec }}\left(k_{3}, k_{1} \ominus k_{2}^{\prime}\right)\right)$. By definition of parallel composition we must have $s_{2} \stackrel{k_{2}^{\prime}}{2} t_{2}^{\prime}$ and $s_{3} \stackrel{k_{3}}{\longrightarrow} t_{3} t_{3}$, and by determinism of $S_{2}, k_{2}^{\prime}=k_{2}$ and $t_{2}^{\prime}=t_{2}$. 